Aus dem anatomischen Institet zu Giessen.

\title{
ÜBER DIE
}

\section{BEDEUTUNG DER OHRMUSCHEL}

\author{
VON
}

B. HENNEBERG, GIESSEN. 

Wenn man von der Funktion der Ohrmuschel spricht, so pflegt man im allgemeinen nur an ihre Bedeutung als Schalltrichter zu denken. Für den Menschen ist diese bekauntlich keine sehr grosse, wohl aber spielt die Ohrmuschel in dieser Beziehung bei vielen Säugetieren eine hervorragende Rolle, wie sich dies schon aus dem lebhaften Spiel der Ohren vieler Tiere erweist. Die Ohrmuschel dient weiter auch als Mittel zum Aus. druck von Gemütsbewegungen. Es sei an den Hund erinnert, der seine Aufmerksamkeit durch das Spitzen der Ohren anzeigt, an das Pferd, das die Ohren zurücklegt, wenn es etwas böses im Schilde führt, und an den Elephanten, der im Zorn seine Ohren aufrichtet. Auf alle diese Verwendungsarten soll an dieser Stelle nicht eingegangen werden, vielmehr soll hier die Bedeutung der Ohrmuschel erörtert werden, die sie als Verschlussapparat für den äusseren Gehörgang besitzt, eine Funktion, die, wie gezeigt werden soll, viel verbreiteter ist, als man je angenommen hat, und durch die die Gestalt der Ohrmuschel erst verständlich wird.

Dass einzelne Tiere imstande sind, ihre Ohren zu verschliessen, ist seit langem bekannt. So weiss man dies von dem Galago, der Ohrenfledermaus, den Pinnipediern und dem Maulwurf. Auch wird erwähnt, dass der Bieber und der Fischotter ihre Ohren zu schliessen vermögen.

Meine Untersuchungen über die Verschliessbarkeit der Ohrmuschel habe ich in der Weise vorgenommen, dass ich eine Anzahl ron Tieren, deren ich leicht habhaft werden konnte, im 
Laboratorium untersuchte. Über diese kann ich demzufolge genaue Angaben machen und auch von einigen Abbildungen liefern. Eine grössere Anzahl wurde in den zoologischen Gärten von Hamburg, Berlin und Frankfurt beobachtet. Ins Einzelne gehende Untersuchungen waren bei diesen leider nicht möglich. Die im Laboratorium untersuchten Tiere wurden aufgebunden und vermittels des Induktionsstromes, durch Anblasen oder durch irgend welche mechanische Reize veranlasst, ihre Ohrmuscheln zu schliessen. Nur der Hamster wurde während des Winterschlafes und beim Erwachen beobachtet. Der Vorgang des Ohrschlusses wurde dann in seinen verschiedenen Stadien beobachtet und bei einigen Tieren photographisch bei Blitzlicht aufgenommen. Um weiter die Schlussfähigkeit zu prüfen, wurden die Tiere in Wasser gebracht und zum Tauchen genötigt. Dann wurde untersucht, ob und wie weit das Wasser in die Ohrmuschel eingedrungen war.

Wir geben nun zuerst im einzelnen die eingehenderen Beobachtungen wieder, die wir an unseren Versuchstieren im Laboratorium angestellt haben, dann kurz (kleingedruckt) diejenigen, die in zoologischen Gärten und Museen gemacht wurden. Darauf werden wir die Ergebnisse unserer Beobachtungen zusammenfassen und endlich ihre Bedeutung für die menschliche Ohrmuschel erörtern.

Bei den eingehender untersuchten Tieren handelt es sich um 1 Raubtier und 2 Insektivoren, sonst nur um Nager, was seinen Grund darin hat, dass letztere uns reichlicher zur Verfügung standen. Trotzdem glauben wir unsere Beobachtungen, auch wenn sie sich auf Repräsentanten derselben Familie beziehen, ausführlicher wiedergeben zu sollen, da jene z. T. sehr verschieden gestaltete, z. T. ähnliche, aber doch in Einzelheiten abweichende Ohrmuscheln besitzen, die clementsprechend einen verschiedenartigen Mechanismus aufweisen. 
Grosses Wiesel (Putorius ermineus'.

Die Concha ist im Verhältnis zur Scapha ${ }^{1}$ ) sehr geräumig. Der Conchaeingang ist weit, sodass ein grosser Teil des Conchabodens freiliegt. Tragus und Helix sind äusserlich nicht von einander abgegrenzt. Die Crista anthel. anterior :) ist gross, lappenförmig. Der Anthelixstamm ist nur als Umbiegungsfalte vorhanden. In beiden finden sich knotenförmige, mit Haaren besetzte Verdickungen. Die Incisura intertrag. ist scharf kreisförmig begrenzt. Der Porus liegt in dem ventralwärts ausgebuchteten Cavum. Die Scapha ist klein. Die Basis ihres oralen Randes setzt sich eine Strecke auf den Kopf zu fort. Der aborale Rand weist eine grosse Hauttasche auf.

Bei elektrischer Reizung während der Narkose erfolgt Schliessung der Incisura intertragica. Dabei legt sich der Antitragus gegen den Conchaboden. Auf solche Weise ist das Cavum nach aussen abgeschlossen, sodass kein Wasser hinein gelangen kann. Der Conchaeingang verengert sich durch Wirkung der Anthelix nur wenig, wenn auch deutlich.

\section{Igel (Erinaceus europaeus).}

Das Auffallendste an dem äusseren Ohr des Igels ist der fast ebene, sehr ausgedehnte Conchaboden, der, da Helix ascendens und Tragus weit von einander entfernt bleiben, durch eine flächenhafte Incisura tragohelicina in die Wange übergeht. An

1) Wie wir in unserem Beitrag über die Entwickelung der Ohrmuschel (1. c. p. 110) bemerkt haben, verstehen wir unter der Scapha nicht nur die konkave Innenfü̈che des freien Abschnittes der Ohrmuschel, sondern das ganze Gebilde von der Anthelix bis zum freien Rande, sodass wir von einer Innen- und Aussenfläche, den Rändern und der Spitze der Scapha sprechen.

-j Wir schliessen uns mit dieser Bezeichnung Schwalbe an (1. c. p. 12:), 1:98). Seine Crista anthel. inferior nennen wir Plica antitragica. 
Stelle des Anthelixstammes findet sich eine Umbiegungsfalte. Unter ihr erstreckt sich die Concha noch ein Stück kaudalwärts. Die Crista anthel. ant. besteht aus einem lappenförmigen, am freien Rande verdickten Gebilde, das, wie der Querschnitt zeigt, eine Falte des Ohrknorpels vorstellt. Sie ist spärlich mit borstenähnlichen Haaren besetzt. Die Anthelixstammfalte geht ohne Bildung einer Plica antitragica in die Begrenzung der Incisura intertragica über. Ebensowenig tritt der Tragus hervor. Hinter ihm liegt der Porus. Die Scapha ist bedeutend breiter als lang, ihr freier Rand rundlich. Die Helix asc. ist kurz. Die Helix setzt sich nicht auf den freien Rand der Scapha fort.

Da die Ohrmuschel, schon wenn der Igel eben erst beginnt sich zusammen zu rollen, unter der stacheltragenden Rückenhaut verschwindet, so wurde das Tier narkotisiert und aufgebunden. Auf elektrische Reizung erfolgt ein vollkommener Ohrschluss. Es macht den Eindruck, als würde die Ohrmuschel in die Tiefe gezogen, was dadurch geschieht, dass sich der beim Igel gewaltig ausgebildete Rückenhautmuskel zusammen mit der Haut von hinten her über den proximalen Teil der Ohrmuschel herüber schiebt und diese nach vorn und gegen den Conchaboden drückt. Der distale Teil der Scapha steht dabei quer $a b$, ist der Länge nach gefaltet und sieht wie eine kleine vollständige Scapha aus. Auf solche Weise. ist die Concha rollständig bedeckt und geschlossen.

\section{Spitzmaus (Crocidura und Sorex).}

Die Ohrmuschel der Spitzmäuse ist dadurch ausgezeichnet, dass sie oralwärts weit offen ist, indem zwischen Tragus und kranialem Ansatz der Scapha ein grösserer Zwischenraum bleibt. Die Incisura intertrag. ist gut ausgebildet. Die grosse schalenförmige Plica antitrag. bildet mit der Scapha eine grosse Tasche. Eine zweite schalenförmige Kulisse findet sich an der Grenze 
zwischen Concha und Scapha und bildet mit dieser eine Tasche. Der freie Rand der Plica ant. und der genannten Kulisse ist mit ziemlich langen Haaren besetzt.

Der Ohrschluss bei unseren einheimischen Spitzmäusen ist ein sehr vollkommener. Die Incisura intertrag. kann vollständig geschlossen werden. Die sehr grosse Plica antitrag. legt sich gegen den Boden der Concha. Bei verschiedenen Versuchen gelangte das Wasser nicht bis an den Meatus acust. externus.

Haselmaus (Iuscardinus avellanarius).

Das Ohr der Haselmaus ähnelt dem der weiter unten näher beschriebenen Nager (Hamster, Ratte. Waldmaus), doch ist es durch eine Antitragustasche ausgezeichnet. Diese kommt dadurch zu stande, dass der aborale freie Rand der Scapha nicht nach dem geschlossenen unteren Ende der Incisura ausläuft, sondern nach dem oberen, und dass er sich etras nach dem Ohrinnern umschlägt. Zwischen diesem Teil der Scapha und der gut ausgebildeten Plica antitragica entsteht die genannte Tasche.

Schon durch leichte mechanische oder akustische Insulte gelingt es, das Tier zum Schliessen seiner Ohrmuschel zu reranlassen. Dasselbe geschieht während res Winterschlafes. Der Ohrschluss ist ein recht vollkommener. Ein schmalerer oraler Teil der Scapha legt sich unter Bildung eines Kiels auf den breiteren aboralen. Beim Schluss der Concha bleibt nur ein T-förmiger Spalt. Der vertikale Schenkel des $T$ entspricht der verengerten Inc. intertrag., der horizontale dem zwischen der Anthelix, die eine gerade Leiste bildet, einerseits und dem Crus hel. und der Plica antitrag. andererseits bestehen bleibenden schmalen Spalt. Die Plica funktioniert nicht als ganzes, sondern als zweigeteiltes, indem sie sich faltet. Das ventrale Stück der Falte beträgt ungefähr zwei Drittel, das dorsale ein Drittel. Die Grenze zwischen 
beiden ist auch an der offenen Ohrmuschel an einer stärkeren Biegung des freien Randes der Plica antitrag. zu erkennen. Der Gipfel der genannten beim Schluss entstehenden Falte legt sich in die Antitragustasche. Der Antitragus und der rentrale Abschnitt der Plica legt sich gegen den Porus ac. ext. und gegen eine Falte, die über dem Porus entlang geht. Letzterer wird dadurch abgeschlossen. Über die Bedeutung des Ohrschlusses während des Winterschlafes ist weiter unten näheres gesagt.

Tatera hirtipes.

Die Ohrmuschel ähnelt in der Ruhe der der Muriden. Wie bei diesen bildet die Scapha den grössereu Abschnitt. Sie ist abgerundet und zeigt nur eine schmale, kurze Helix, die sich gegen die Scapha nicht deutlich abgrenzt. Die Helix ist mit ziemlich langen gleichmälsig kaudalwärts gerichteten Haaren besetzt. Der Eingang in die Concha wird durch eine kulissenartig vorspringende Crista anthel. ant. begrenzt. Eine Anthelixstammfalte tritt nicht sehr stark hervor. Der Antitragus springt wulstförmig nach aussen vor und ist auf seiner Aussenfläche und an seiner Kante mit borstenähnlichen Haaren besetzt. Der Tragus ist schwach ausgebildet, tritt nicht stark hervor und verläuft sich nach imnen von der Helix in die Concha hinein und grenzt so die Cymba rom Carum ab. Die Incisura intertrag. ist mälsig weit, an ihrem geschlossenen Ende gleichmälsig gerundet. Die laterale Wand des Cavum ist blasenförmig aufgetrieben, der kraniale Rand des Porus ist ron einer breiten Hautfalte begrenzt.

Auf Anblasen oder auf zirpende Geräusche hin legt das Tier seine Ohrmuschel nach hinten und bedeckt mit dem oralen Jängsabschnitt der Scapha, der ungefähr ein Drittel der ganzen ausmacht, wobei eine scharfe Kielung der Scapha eintritt, den aboralen breiteren Längsabschnitt. Dabei bleibt 
nur der ventrale Teil der Incisur sichtbar, sonst ist der Eingang in die Concha vollständig bedeckt. Über die Incisur und auf den aboralen Längsabschnitt der Scapha legen sich die langen am oralen Rand der Scapha stehenden Haare. Oft wird das distale Drittel der Scapha nach dem Kopf hin etwas eingekrempelt. Öffnet man die Scapha, so sieht man, dass der Conchaschluss ein ausserordentlich vollkommener ist. Die Crista anthel. ant. springt als breite Kulisse vor, sodass sie bis an den Tragus heranreicht, und hat sich fest gegen den Boden der Concha gelegt. Ebenso verhält sich eine stark vorspringende Falte, die sich an Stelle des Anthelixstammes gebildet hat. Diese liegt ohrinnenwärts von der Crista anthel. ant.. Nach aussen von der letzteren rerläuft eine scharfe quer zur Längsachse der Scapha liegende Falte. Die Incisur ist vollständig geschlossen, der nach aussen wulstförmig vorspringende Antitragus hat sich ebenfalls fest gegen den Conchaboden gelegt, sodass das Carum conchae vollständig abgeschlossen ist. Öffnet man die Incisur, so beobachtet man, dass die den dorsalen Rand des Porus umsäumende breite Hautfalte sich gegen die gegenüberliegende Cavumwand gelegt hat, sodass auf solche Weise hier nochmals ein Abschluss erzeugt wird. Ich nehme an, dass das Ohr geschlossen wird, wenn das Tier gräbt. Es wird dadurch verhindert, dass der Sand in das Ohr gelangt. Die Haare an der Helix wirken in demselben Sinne.

Hamster (Cricetus frumentarius).

(Taf. 7, Fig. 1--3).

Die Ohrmuschel des Hamsters ähnelt in Ruhestellung (Fig. 1) ganz ausserordentlich der der Ratte und der Waldmaus, sodass, da jene genau geschildert werden sollen, wir von einer eingehenden Beschreibung absehen können. Während aber hei der letzteren der freie Rand des Tragus in den der Helix direkt übergeht, 
verläuft sich beim Hamster der Tragus nach innen ron der Helix wie bei der Ratte. Dagegen hat der Hamster mit der Waldmaus die stärkere Ausbildung des Carum conchae gemeinsam, dessen laterale Wand sich blasenförmig vorwölbt. Wie bei der Waldmans setzt sich auch der Antitragus in eine kulissenartige Plica antitragica fort, die also aus einer wirklichen Knorpelplatte besteht.

Während des Winterschlafes (Fig. 3) ist die Ohrmuschel nach hinten gerichtet und an den Kopf angelegt, die Scapha ist in der Längsrichtung ziemlich scharf gekielt, wodurch ein schmialerer oraler und ein breiterer aboraler Abschnitt entsteht. In der Breite verhalten sich diese Teile wie $1: 2$. Der orale Abschnitt, an clem die Helix nicht mehr als ein besonderer Teil sichtbar ist, legt sich dicht auf den aboralen auf und beleckt diesen derart, dass nu die aborale stärker behaarte Hälfte sichtbar bleibt. Auch von der Incisura intertragica ist nur die untere Partie sichtbar. Der aborale breitere Teil zeigt zwei stark ausgebildete Querfalten, die in ihrer ganzen Ausdehnung sichtbar werden, sobald man das orale Drittel emporklappt oder wenn man eine nicht so fest geschlossene Ohrmuschel betrachtet (Fig. ?). Die proximale Querfalte legt sich gerade noch über den Spalt zwischen Anthelix und Plica antitragica herüber. Die distale geht von dem aboralen Rande der Scapha aus an der Grenze zwischen den beiden basalen Dritteln und dem distalen Drittel. Sie erstreckt sich bis zu dem Längskiel. An ihrem Anfang ist sie höher. nach dem Längskiel hin wird sie niedriger. Der zwischen cliesen beiden Falten gelegene Abschnitt der Scapha zeigt ein oder zwei geringer ausgebildete Falten oder Wülste. Durch diese Querfalten wird die Scapha in der Längsrichtung nicht unbedeutend rerkürzt.

Der Eingang in die Concha ist ganz bedeutend verengert. Er wird begrenzt durch die Anthelix, Plica intitragica, Antitragus. Tragus, Nurzel der Helix. Die Verengerung tritt da- 
durch ein, dass die Anthelix stark hervortritt und dass sie und der Antitragus sich einander nähern. Dies kann bis zu dem Grade geschehen, dass Plica ant. und Anthelix einander parallel laufen und nicht mehr als $1 / 2 \mathrm{~mm}$ von einander entfernt sind. Auf solche Weise erstreckt sich die Cymba hinter der Anthelix noch ein Stück kranialwärts. Die vorspringende Anthelix hat sich dem Boden der Concha genähert. Dasselbe gilt auch von dem Antitragus und der Plica antitragica. Dadurch wird die Incisura intertragica stark verengert. Der hinter dem Antitragus und weiter ventral liegende Raum, Cavum conchae, nimmt ebenfalls bedeutend an Ausdehnung ab, indem sich seine laterale Wand der medialen genähert hat.

Die ganze Ohrmuschel verschwindet auf solche Weise fast vollständig im Pelz und zwar dadurch, dass sie sich verkürzt, dass sie sich dem Kopfe anlegt, 'und dass die harfreien Abschnitte durch das Herüberlegen des oralen Drittels bedeckt werden. $\ddot{C}$ ber den unteren Abschnitt der Incisura intertrag. legen sich die auf der Wange stehenden Haare herüber. Die Aussenfläche des oralen Längsdrittels ist mit Haaren bedeckt, die in der Farbe und Länge mit den Haaren der nächsten Umgebung übereinstimmen. Auch auf das aborale Drittel legen sich die Nachbarhaare auf. Dass die unbehaarte Aussenseite der Scapha bedeckt ist, ist selbstverständlich, da sie sich an den Kopf seitlich anlegt. All dies ist auf unseren Figuren nicht zu sehen, da die Haare in der Umgebung des Ohres weggeschoren wurden, damit die Ohrmuschel sichtbar bliebe.

Über clie Bedeutung der Zusammenfaltung und des Schlusses der Ohrmuschel während des Winterschlafes siehe weiter unten. Bemerkenswert ist, dass dabei die unbehaarten Teile der Ohrmuschel bedeckt werden. Auch beim gewöhnlichen Schlaf tritt bein Hamster eine Verengerung des Conchaeingangs ein. Ein Scaphaschluss erfolgte bei unserem Versuchstier hierbei nicht. Möglich ist jedoch, dass das Tier, wenn es vollständig ungestört 
gewesen wäre, auch die Scapha wenigstens etwas geschlossen hätte. Beim Schwimmen tritt ebenfalls der Ohrschluss ein und bleibt auch noch einige Zeit, nachdem das Tier aufs Trockene gelangt ist, bestehen. Bei unserem Versuchstier wurde jedoch verschiedene Male beobachtet, dass beim Tauchen Wasser in die Concha eindrang. Es scheint also, als ob der Schlussmechanismus dem Eindringen des Wassers gegenüber beim Hamster nicht sehr prompt funktionierte. Durch Schütteln des Kopfes schleudert das Tier die Wassertropfen leicht von der fettigen Haut der Concha ab. Kratzt sich das Tier an der Wange, so legt es die Ohrmuschel derselben Seite nach hinten und schliesst sie. Es handelt sich dabei wohl um eine gleichzeitige zwecklose Innervation der Ohrmuskulatur.

\section{Wanderratte (Mus decumanus).}

(Taf. 7, Fig. 4-6.)

Die Morphologie des Rattenohres (l. c. p. 111) haben wir an anderer Stelle geschildert, so dass wir auf jene Darstellung verweisen können. Das Rattenohr in Ruhestellung zeigt Fig. 4.

Bei Reizung z. B, durch den Induktionsstrom wird die ganze Ohrmuschel kaudalwärts zurückgelegt. Der orale Längsabschnitt der Scapha bis zum Längskiel legt sich auf den aboralen breiteren Längsabschnitt. Dabei kommt die abgerundete Spitze der Helix auf die Scapha zu liegen und zwar dort, wo der Antitragus in die Plica antitragica übergeht (Fig. 6). Bei stärkerer Reizung krempelt sich die ganze distale Querhälfte der Scapha nach ihrer Aussenseite hin um und schmiegt sich dem Kopfe an, wobei eine stark vorspringende proximale Querfalte entsteht. Die distale Querhälfte der Scapha zeigt zwei bis drei Querfalten. Der distale Teil des herübergeklappten oralen Längsabschnittes macht die Beregung der distalen Querhälfte mit. Es ist dies der Scaphaschluss. Auf solche Weise wird die Concha 
und der grösste Teil der Incis. intertrag. vollständig bedeckt. Um diese sichtbar zu machen, muss man den oralen Längsabschnitt mit der Pinzette zurückklappen (Fìg. ja u. b). Daun zeigt sich, dass die Incis. intertrag. vollständig geschlossen ist dadurch, dass sich Tragus und Antitragus dicht aneinander gelegt haben.

Der weitere Schluss der Ohrmuschel kann auf zweierlei verschiedene Weise geschehen. In dem einen Falle (Fig. ja) treten Crista anthel. anterior und Plica antitrag. stark herror und nähern sich einander fast bis zur Berührung, sodass sie fast parallel nebeneinander liegen oder einen sehr spitzen Winkel mit einander bilden. Dies geschieht, wie die Beobachtung zeigt, indem die Crista anthel. ant. nach der Plica antitrag. hin und letztere nach der ersteren zu bewegt wird. Der Scheitel des Winkels ist gegen die proximale Querfalte gerichtet. Im andern Falle (Fig. 5 b), den man hauptsächlich bei elektrischer Reizung beobachtet, nähern sich Crista anthel. und Plica autitrag. nicht so stark, sondern bilden einen weniger spitzen Winkel miteinander. Zwischen den Schenkeln dieses Winkels liegt ein Wulst, der sich jenen fest anschmiegt. Gebildet wird derselbe dadurch, dass sich der Boden der Concha gewulstet hat. Er hat die Form eines Dreiecks. Der spitze Gipfelwinkel desselben wird von dem Anthelix-Plicawinkel gebildet, die Seiten werden von der Crista anthel. und der Plica begrenzt, die Basis liegt hinter der Helix ascendens und an dem Teil des Tragus, der sich in die Concha hineinbiegt. - In beiden Fällen pressen sich Anthelix und Plica antitrag. fest gegen den Boden der Concha. Auf solche Weise entsteht ein geschlossener Cymba- und Carumraum. Beide Räume kommunizieren nicht oder doch nur an dem genannten spitzen Winkel miteinander. Der Cymbaraum wird von der kulissenartig rortretenden Crista anthel. und dem Boden der Crmba gebildet. Er ist durchaus spaltförmig. Der Carumraum erstreckt sich hinter die geschlossene Incisura inter- 
tragica. Er wird begrenzt von der Plica antitragica, Antitragus, Tragus und Boden des Cavum. Er ist, da sich die Plica, Tragus und Antitragus gegen den Boden des Cavum legen, ebenfalls spaltförmig.

Durch den Schluss der Incisura und die eben genannte enge Anlagerung des Tragus und besonders des Antitragus wird auch der Porus direkt geschlossen. Dabei spannt sich die dünne Hautfalte, die sich an dorsalen Rande des Porus findet, an und legt sich gegen den Antitragus. Auf solche Weise erfolgt der Porusschluss.

Die Ratte schliesst ihre Ohrmuschel auf mechanische, akustische oder elektrische Reizung. Ein vorzüglicher Ohrschluss tritt auf, wenn man das Tier tauchen lässt. Bekanntlich ist die Ratte ein guter Schwimmer und Taucher. Oft durchbricht hierbei das Wasser nicht einmal den Scaphaschluss. Nur wem das Tier stark irritiert wird, dringt das Wasser bis in das Cavum. Sobald das Tier aus dem Wasser herauskommt, schüttelt es heftig den Kopf, um das anhaftende Wasser: abzuschleudern. Hierbei entfaltet und richtet es die Ohrmuschel auf. Verhindert man das Tier am Kopfschütteln, wie es gelingt, wenn man es noch im Wasser im Genick fasst, so lässt es auch die Ohrmuscheln noch längere Zeit geschlossen. Man hat damn Gelegenheit, den Schlussmechanismus zu beobachten und kann bequem feststellen, ob und wie weit Wasser eingedrungen ist.

Waldmaus (Mus sylraticus.)

(Taf. 7, Fig. 7. Taf. 8, Fig. 8 и. 9.)

Die Ohrmuschel der Waldmaus (Fig. T) ist ebenso gross wie die der Ratte. Es hat also die Waldmaus eine relativ grössere Ohrmuschel als jene. Die grösste Breite der Scapha beträgt $14 \mathrm{~mm}$, ihre Länge auf der Aussenseite gemessen $16 \mathrm{~mm}$. Die 
Ohrmuschel verdankt also ihre Grösse der bedeutenden Ausdehnung der Scapha. Aber auch die Concha ist weit und geräumig. Die Scapha hat die Form eines vorn stark abgerundeten verhältnismä fsig breiten Löffels. Sie wird von der Concha durch eine Crista anthel. ant. und eine Plica antitragica abgegrenzt. Eine Spitze fehlt. Ihr Rand ist vielmehr gleichmäfsig gerundet. Sie ist aussen und innen kurz behaart. In der Ruhe ist die Scapha in der Querrichtung ziemlich gleichmäfsig gerundet, in der Längsrichtung fast gerade. Ungefähr die proximale Hälfte des oralen Randes ist nach dem Ohrinnern ungeschlagen und bildet die Helix. Die grösste Breite derselben beträgt $1 \mathrm{~mm}$. Sie beginnt als Helix ascendens an der Incisura intertrag., die sie äusserlich oralwärts begrenzt. Die Concha sitzt breit dem Kopfe an. Sie zeigt einen weiten Eingang, der durch Crista anthel., Plica antitragica, Incisura antitragica und Helix begrenzt wird. Dieser direkt sichtbare Abschnitt stellt die Cymba vor. Das Carum wird von einer ventralen Ausbuchtung des vor dem Porus acusticus gelegenen Abschnittes der Concha gebildet. Es ist relativ geräumiger als bei der Ratte. Die Anthelix ist nur als eine Crista anthelicis anterior vorhanden, indem sie eine deutlich nach dem Ohrmuschelimem hervorspringende Falte bildet, die hinter der Helix vorkommend, niedriger werdend bis zur Längsmitte der Scapha verläuft. Die Plica antitragica stellt eine stark vorspringende flach bogenförmig begrenzte Platte vor, die ohne Grenze in den Antitragus übergeht, der dorsalwärts die Incisura intertragica begrenzt. Crista anthel. und Plica antitrag. sind also bei der Waldmaus stärker ausgebildet als bei der Ratte. Der Tragus tritt am unversehrten Objekt kaum als solcher hervor. Untersucht man den freigelegten Ohrknorpel, so sieht man, dass er sich nach innen in den Muschelgrund fortsetzt und auf solche Weise die äusserlich wenig markant hervortretende Grenze zwischen der Cymba und dem Carum bildet. Die 
Incisura ist weit, unten flach bogenförmig begrenzt. Das Cavum erstreckt sich ziemlich weit ventralwärts.

Bei starker Reizung durch den Induktionsstrom wird die Ohrmuschel (Fig. 8) nach hinten gelegt, sodass die Längsachse derselben kraniokaudal verläuft. Dabei liegt der distale freie Rand derselben dem Körper an. Das orale Längsviertel der Scapha ist nach der Innenfläche der Scapha herüber geklappt, sodass es mit dem grösseren Abschnitt der Scapha einen Kiel bildet. Der genannte orale Abschnitt verdeckt auf solche Weise den Eingang in die Concha vollständig, sodass nur das unterste Ende der Incisura intertrag. sichtbar bleibt. Der grössere aborale Längsabschnitt der Scapha zeigt eine bis drei quer zu seiner Längsrichtung verlaufende Falten in der Gegend seiner Quermitte. Hier biegt sich der grössere distale Teil der Scapha nach dem Halse des Tieres hin. Von den genannten Querfalten ist die proximale, nach der Concha hin gelegene bei weitem am stärksten ausgebildet. Sie springt mit einer scharfen Kante stark hervor. Diese freie Kante ist bogenförmig, mit der Konkavität oralwärts gerichtet. Gegen diese nach aussen konvex vorspringende Faltenpartie legt sich der orale herübergeklappte Längsabschnitt der Scapha. Der freie Rand dieses Abschnittes legt sich mehr oder weniger eng über die Incisura herüber und auf den Teil der Scapha, von dem die Plica antitragica sich erhebt. Auf diese Weise entsteht unter dem herübergeklappten oralen Längsabschnitt der Scapha eine Kammer (Scaphaschluss).

Hebt man den oralen Längsabschnitt empor oder unterbleibt der Scaphaschluss (Fig. 9), so sieht man ein von der Ruhelage der Ohrmuschel stark abweichendes Bild. Bei stärkster Kontraktion der Ohrmuskulatur finden wir folgendes. Ein Einblick in die Concha ist jetzt nicht mehr möglich. Die Crista anthel. springt mit der angrenzenden Partie der Scapha und der Cymba kulissenartig von oben her ausserordentlich stark vor, von unten her hat sich die Plica antitrag, die jetzt die Gestalt einer halb- 
kreisförmigen Scheibe angenommen hat, jener Kulisse entgegen geschoben, sodass sich der freie gradlinige Rand der Anthelixfalte und die kreisförmige Peripherie der Plica antitragica berühren wie eine Tangente einen Kreisabschnitt (Anthelixschluss). Kranial und kaudal von der Berührungsstelle blejbt je eine minimale Ötfnung, die in den Concharaum führt. Die kaudale wird kaudal. wärts durch eine stark vorspringende Falte begrenzt, die ohrinnenwärts von der Anthelixfalte und der Plicascheibe liegt und von dem distalen Ende der ersteren nach der letzteren hin verläuft und jetzt eine scharfe Abgrenzung zwischen Concharaum und Scapha bildet. Diese Falte ergänzt also die Anthelix und vertritt den Stamm der Anthelix, wie er sich bei vielen Tieren auch schon in der Ruhestellung und beim Menschen als unbewegliches Gebilde stets findet. Wir haben sie daher Anthelixstammfalte genannt. Die kraniale der genannten beiden Öffnungen geht in die Incisura intertragica über. Die Incisur hat sich sehr stark verschmälert, dadurch, dass sich die Plica antitragica, Tragus und Helix ascendens einander fast bis zur Berührung genähert haben. Auf solche Weise ist der Cymbaraum nach aussen hin bis auf die genannten kleinen Öffnungen vollständig abgeschlossen. Er liegt hinter Anthelixkulisse, der Stammfalte und der Plicascheibe, wo er in den Cavumraum übergeht. Da sich die genannten Falten gegen den Boden der Cymba drücken, so ist der Cymbaraum nur spaltförmig. In dem von uns wiedergegebenen Stadium (Fig. 9) war es durch die Stellung der Elektroden erreicht, dass der Cymbaschluss allerdings nicht vollständig - wie der zu grosse Abstand zwischen Anthelix und Plica antitragica und die Grösse der Incisur zeigen - erfolgte, ohne dass sich die Scapha herüber geklappt hätte. Auf solche Weise werden Crista anthel. und Plica antitragica in ihren Lagebeziehungen und in der Tiefe auch die Anthelixstammfalte sichtbar - Der Cavumraum bleibt auch bei starker Kontraktion geräumiger, indem sich mediale und laterale Wand nicht so eng 
aneinander legen. Er wird abgeschlossen durch die Plicascheibe, die sich gegen den Boden des Cavums gelegt hat, und durch den Schluss der Incisura intertragica. Der Porus acusticus ext. ist dadurch, dass die Hautfalte an seinem dorsalen Rande stärker vorspringt, verkleinert.

Man kann die Waldmaus auf verschiedene Weise veranlassen, ihre Ohrmuschel zu schliessen. Auf Kitzeln, Anblasen, Geräusche z. B. auf Pfeifen, mehr noch auf Zirpen mit den Lippen und am besten auf Quietschen, wie man es durch Drehen eines Glasstöpsels im Flaschenhals erzeugen kann, reagiert sie prompt. Bei der Herstelluug der photographischen Aufnahmen wurde der Induktionsstrom benutzt. Ein vorzüg. licher Ohrschluss findet beim Schwimmen statt, worin die Waldmaus recht geschickt ist. Dabei legen sjch die vor dem $\mathrm{Ohr}$ stehenden Haare über die Ohrmuschel. Sie spielen aber keine grosse Rolle, da auch bei Tieren, denen man jene Haare abgeschnitten hat, das Wasser nicht eindringt. Fast stets ist die Concha auch nach mehrfachem Tauchen absolut trocken. Auf der behaarten Innenfläche der Scapha haftet nur wenig Wasser, das bald abgeschleudert oder abgeputzt wird. Dieses Wegschleudern des Wassers, was durch eine schnelle Schüttelbewegung des Kopfes vorgenommen wird, besorgen übrigens nicht alle Waldmäuse, offenbar da es nicht nötig ist.

\section{Brandmaus (Mus agrarius).}

Die Ohrmuschel der Brandmaus ähnelt sowohl durch ihre geringe Grösse wie auch sonst der der Feldmaus. Von Abweichungen sei erwähnt, dass bei der Brandmaus eine leistenförmig hervortretende Crista anthel. ant. vorhanden ist. Der Ohrschluss erfolgt z. B. auf mechanische und akustische Reize. Es tritt ein guter Anthelixschluss und ein fast rollkommener Incisuraschluss ein. 
Hausmaus (Mus domesticus).

Die Gestalt der Ohrmuschel der Hausmaus ist so bekannt und bietet auch mit den bereits beschriebenen Mäuseohren verglichen so wenig besonderes, dass von einer Beschreibung abgesehen werden kann. Der Scapha-, Anthelix- und Incisuraschluss ist recht vollkommen und ähnelt dem, wie er von den Gattungsgenossen beschrieben wurde.

\section{Rötelmaus (Hypudaeus glareolus).}

Bei der Ohrmuschel der Rötelmaus ist von Besonderheiten nur die Grösse der ungefähr halbkreisförmigen Plica antitragica zu erwähnen. Sie ist riel grösser als die der übrigen beschriebenen Vertreter der Gattung Mus mit Ausnahme der Zwergmaus. Die Anthelix, die als eine Crista anthel. anterior corhanden ist, besteht nur aus einer in der Ruhestellung wenig ausgesprochenen Cmbiegungsfalte. Die Falte, die den Por. ac. ext. an seinem aboralen Umfange begrenzt, ist sehr reichlich. Auf der Helix ascendens, dem Tragus und Antitragus finden sich lange Haare, die sich über den Conchaeingang legen. Die distale Hälfte der Scaphainnenfläche ist kurz behaart. Im übrigen ist die Innenfläche der Ohrmuschel unbehaart.

Reizung mit dem Induktionsstrom zeigt, dass die Rötelmaus ihre Ohrmuschel recht gut schliessen kann. Es tritt eine starke Umkrempelung der Scapha ein, wobei mehrere Falten entstehen. Die Anthelix tritt stark hervor. Sie und Plica antitrag. nähern sich einander ziemlich bedeutend. Die Incisura int. verengert sich stark, doch kommt es nicht zu einem vollkommenen Schluss. Die Plica antitrag. legt sich gegen den Boden der Concha, der sich vorwölbt und der Plica entgegenkommt. Die Plica wird dabei nach aussen konkav. Die Bedeutung dieser Aushöhlung könnte darin zu suchen sein, dass das Wasser die Plica auf 
solche Weise gegen den Conchaboden drückt. Es handelt sich also um einen passiven Verschluss. Die Umgebung des Porus ac. wölbt sich stark vor und legt sich gegen Tragus und Antitragus, wie überhaupt gegen die laterale Wand des Cavum. Hierdurch kommt es zu einem guten Abschluss des Porus. Zufällig wurde das Verhalten der Ohrmuschel dem Eindringen des Wasser's gegenüber nur am toten Tiere geprüft. Es zeigte sich, dass es nicht einmal einer stärkeren Verengerung des Conchaeinganges bedarf, um das Wasser abzuhalten. Fast stets fing sich eine Luftblase hinter der Plica antitragica, die mit der im Meatus zusammenhing. Die nackten Teile der Ohrmuschel zeigen eine fettige Oberfläche. Darauf beruht es, dass sich die Luftblasen mit der Pinzette abnehmen und unter Wasser transportieren lassen.

Feldmaus (Arricola arvalis).

Die Ohrmuschel und besonders die Scapha der Feldmaus ist im Vergleich zu der der Waldmaus klein, sodass sie nur wenig aus dem Pelz hervorragt. Die Scapha erscheint fast wie quer abgestutzt. Da sie nur kurz ist, nimmt die Helix fast den ganzen oralen Rand derselben ein. Der Eingang in die Concha wird in seinem ganzen Kontur von der Crista anthel. anterior, Plica antitragica, Antitragus, Incis. intertrag., Tragus und Helix ascendens begrenzt. Die Anthelix ist nichts anderes als eine stärkere Umbiegungsstelle zwischen. Concha und Scapha. Der Antitragus tritt deutlich hervor. Die Plica antitrag. ist mälsig stark entwickelt. Der Tragus verläuft sich nach innen von der Helix in die Concha hinein. Der Porus akust. ist, abgesehen von seinem oralen Rande, von einer scharf vorspringenden Falte umgeben.

Die Concha und die sich anschliessenden Teile der Innen. seite der Scapha sind unbehaart, richtiger mit makroskopisch schwer sichtbaren Haaren besetzt. Ungefähr die distale Hälfte 
der Scapha ist behaart. Der freie Rand des Tragus, auch des Teils, der in die Concha hineinläuft, des Antitragus und der Plica antitragica sind mit borstenähnlichen Haaren besetzt.

Beim Ohrschluss wird die Scapha nach hinten dem Kopf angelegt. Es bildet sich auf ihr an der proximalen Haargrenze eine stark vorspringende sichelförmige Querfalte, deren Konkavität nach der Concha gerichtet ist. Die Helix legt sich an, bedeckt aber nur einen kleinen Teil des Conchaeinganges. Letzterer ist jetzt überall scharfrandig begrenzt. Er hat sich nur so weit verengert, dass die gegenüberliegenden Ränder desselben fast überall $1 \mathrm{~mm}$ roneinander entfernt bleiben. Er hat die Gestalt eines Bogens angenommen. Die Konvexität desselben wird ron der Anthelix begrenzt, die jetzt kulissenartig vorspringt, die Konkarität von dem zungenförmigen Antitragus. Auch die Ränder der Incisura intertrag. haben sich einander etras genähert.

Der Ohrschluss kamn auf dieselben Reize wie bei anderen Iäusen herbeigeführt wercen. Obgleich der Eingang in die Concha ziemlich weit bleibt, funktioniert er ausgezeichnet. Bei zahlreichen Tauchrersuchen - die Feldmaus schwimmt recht gut - blieb die Concha fast stets trocken. Meist gelangt das Wasser also nur bis an den Conchaeingang. Die Luft ist dann hinter der Anthelix, Plica antitragica, Antitragus und Tragus gefangen. In selteneren Fällen dringt das Wasser in die Cymba ein, es wird jedoch die Luft im Cavum hinter Tragus, Antitragus und Plica antitragica festgehalten. Hierbei spielt offenbar der geschilderte Haarbesatz dieser Teile eine Rolle. Der Antitragus legt sich dabei nach dem Conchaboden hin. Wie es scheint, wirkt hierbei der Wasserdruck mit. Es wird dadurch die Entfernung zwischen Antitragus und Plica antitragica und der gegenüberliegenden Conchawand verkleinert, ausserdem wird die Incisura intertragica etwas enger. Versagt auch dieser Mechanismus, was nur sehr selten geschieht, so dringt das Wasser bis an 
den Porus. acust. ext. Dass es in diesen hineingestürzt wäre, wurde niemals beobachtet, da die Luft hier sehr energiseh hinter der beschriebenen Hautfalte am Porus festgehalten wird. Dieser Vorgang ist ein automatischer, der sich an toten Tiere ebenso beobachten lässt, wie am lebenden. Auch von der Wirkungsweise der äussersten Barriere (Conchaeingang) kann man sich oft am frischtoten Tiere überzeugen, wemn die Ohrmuschel noch etwas von der Schlussstellung zeigt. Dabei sieht man, wie sich die vor der Ohrmuschel stehenden Körperhaare über die Luftblase hinweglegen. Da sie sich aber von der letzteren abheben lassen, ohne dass diese platzt, so sind sie offenbar zum Zustandekommen des Abschlusses nicht nötig. Dies zeigt sich denn auch an lebenden Feldmäusen, denen man die Haare vor der Ohrmuschel weggeschnitten hat. Der Cavumabschluss ist auch an toten Tieren leicht zu beobachten. Wegen des gut funktionierenden Ohrschlusses schüttelt die Feldmaus in der Regel nach dem Bade nicht den Kopf, un das Wasser aus den Ohrmuscheln herauszuschleudern. Dass der Boden der Concha, auch wemn Wasser eingedrungen ist, trocken ist oder nur kleine Wasserperlen zeigt, erklärt sich aus dem fettigen Überzug jener 'Teile. - Dass auch bei den übrigen geschilderten Tierohren die eingeschlossene Luft dem Findringen des Wassers gegenüber eine Rolle spielt, ist selbstverständlich. Wir kommen weiter unten darauf zurück.

Meerschweinchen (Caria cobaya).

(Taf. 8, Fig. 10 bis 12.)

Die Ohrmuschel des Meerschweinchens ${ }^{1}$ ) ist in Ruhestellung (Fig. 10) ungefähr ebenso breit wie lang. In ihrer Grösse und äusseren Begrenzung derartig gestaltet, dass sie sich mit einem

1) Fntwicklungsgeschichtliche ['ntersuclungen haben uns veranlasst, im folgenden einzelne Gebilde an der Ohrmuschel des Yeerschweinchens anders zu benennen, als dies z. B. Johannes Schmidt tut. 
Zweimarkstück so bedecken lässt, dass nur am oberen Rande, wo dieser in den kaudalen umbiegt, ein sichelförmiges Stück unbedeckt bleibt. Während die Scapha mit Tragus und Antitragus ein flächenhaftes, ungefähr in einer Ebene liegendes Gebilde vorstellt, bildet die Concha (mit Fossa triang.) eine um einige Millimeter eingesenkte Crube. Diese sitzt fast in ganzer Ausdehnung clem Schädel an. In jener Grube fallen sofort zwei lappenförmige Gebilde auf. Eins derselben grenzt dieselbe gegen die Scapha ab. Es ist dies das Crus anthelicis superius. Dasselbe stellt eine halbkreisförmige, von der Haut überzogene Knorpelscheibe vor, die ungefähr senkrecht auf ihrer Unterlage in der Richtung der Längsachse des Tieres aufsitzt. Ihr freier gebogener Rand ist mit mehreren Reihen borstenälmlicher, nach oben gekrümmter Haare besetzt. Kaudalwärts geht das Crus sup, indem es im abgerundeten rechten Winkel nach oben umbiegt, in einen immer niedriger werdenden, auf der Scapha liegenden Wulst über. Derselbe ist als Plica longitudinalis aufzufassen. Die Behaarung des Crus stip. setzt sich noch ein kurzes Stück auf die Plica long. fort. Von der genannten Umbiegungsstelle an nach unten setzt sicb die Concha gegen die Scapha dadurch ab, dass sich die letztere in der Fläche gegen die erstere abknickt. Es entsteht so eine Art Falte. Diese rertritt den bei vielen Formen vorhandenen Anthelixstamm. Sie wurde daher Anthelixstammfalte genannt. Das andere nach unten von dem Crus sup. gelegene ähnlich gestaltete etwas kleinere lappenförmige Gebilcle ist das Crus anthel. inferius. Wie bei jenem bildet eine einfache Knorpelplatte sein Gerüst. Die Richtung desselben ist die gleiche wie die des ersteren. Es ist nur spärlich mit borstenähnlichen Haaren besetzt. Mit der Anthelixstammfalte steht es in keinem Zusammenhang. Vielmehr kommuniziert die zwischen den beiden Crura gelegene Fossa triang., die einen parallelwandigen Gang vorstellt, zwischen Crus inferius und Stammfalte breit mit der Concha. 
Die Anthelixstammfalte geht nach unten mehr oder weniger deutlich in die untere (ventrale) Begrenzung des Conchaeinganges über. Hier tritt die Incisura intertrag. als ein breiter, nicht sehr tiefer, ausgerundeter Einschnitt deutlich hervor. Der sie begrenzende Tragus und Antitragus zeigen keine ins Auge fallende Formen. Ersterer zeigt einen gradlinigen freien Rand, der oben nach innen $d$. $h$. in die Concha hinein umbiegt und hier bald verschwindet, letzterer einen flach gewölbten. Nach der Aussen- und Innenfläche springt der Antitragus wulstförmig vor. Er ist mit borstenähnlichen Haaren bestanden. Von ihm nach hinten nach der Anthelixstammfalte hin verläuft die Plica antitragica. Hinter Antitragus und Plica ant. erstreckt sich die Concha noch ein Stück ventralwärts, hinter dem Tragus oralwärts. An letzterer Stelle liegt der Porus acust. ext. Der obere Rand desselben wird von einer Hautfalte begrenzt.

Die Scapha, deren Gestalt schon beschrieben wurde, besitzt eine Helix, die den vorderen Scapharand eimnimmt. Sie schliesst sich an den 'Tragus an, berührt das orale Ende des Crus anth. inf. und bedeckt dieselbe Strecke des Crus anth. sup. Sie ist auf der Aussenseite behaart, innen kahl. Die Scapha ist auf der ganzen Innenfläche d. h. auf der nach dem Porus führenden Fläche behaart mit Ausnahme des zwischen Helix und Crus anth. sup. liegenden Teiles und der sich nach hinten nach dem freien Rand der Seapha hieran anschliessenden Partie. - Somit wird der Eingang in die Concha begrenzt durch das Crus anth. inf., die Anthelixstammfalte, Plica antitragica Antitragus (Incisura intertrag.) Tragus und Helixwurzel. Die Concha ist geräumig. Sie hat ungefähr ein Drittel der Grösse der Scapha. Eine Tremung in Cymba und Carum ist durch den nach innen einbiegenden Teil des Tragus angedeutet. Fossa triangularis und Concha sind frei von Haaren.

Das Meerschweinchen ist im Stande, durch Kontraktion seiner Ohrmuskeln die Gestalt der Ohrmuschel mehr oder 
weniger stark zu verändern, wie der Vergleich der Abbildungen zeigt. Das Tier tut dies, wenn es in Angst ist, wenn es ins Wasser gesetzt wird, oder wenn das Ohr durch Anblasen, Kitzeln oder Elektrisieren gereizt wird. Für die photographischen Aufnahmen wurde der letztere Weg gewählt. Da man aber hier je nach der Lage der Elektroden und der Stärke des Stromes verschiedene Bilder bekommt, da einmal diese, das andere Mal jene Muskeln sich stärker kontrahieren, so war es nötig, mehrere Aufnahmen zu reproduzieren, Es gelingt zuweilen, sämtliche Muskeln gleichzeitig zur Kontraktion zu bringen, doch war es mir nicht möglich, solchen Zustand auf die Platte zu bringen.

Auf Reizung mit dem Induktionsstrom wird die Scapha nach hinten dem Kopfe angelegt und die Ohrmuskulatur in Tätigkeit gesetzt. Die Ohrmuschel lässt jetzt vier mehr oder weniger abgeschlossene Räume oder Kammern entstehen, die einzeln geschildert werden sollen.

1. Die Helixkammer. (Fig. $11 \mathrm{a}$, b.) Die Helix nähert sich dem zwischen ihr und dem Crus anthel. sup. liegenden Abschnitt der Scapha und zugleich dem Crus anthel. sup. und der Plica longitud. Das unter der Helix liegende Stück des Crus anthel. sup. legt sich fest gegen die Helix. So entsteht eine Art Düte. Wandung und Begrenzung derselben wird gebildet von der Helix, dem Crus anthel. sup., dem zwischen beiden liegenden Teil der Scapha und der Plica longitudinalis. Die auf dem Crus anthel. sup. angeordneten Haare sind nach oben also derart gerichtet, dass sie die Wandung der Düte vervollständigen helfen. Ebenso tragen die auf der Plica long. stehenden Haare zum Verschluss der Helixkammer bei. Auf die Lücke zwischen Helix und Crus anthel. sup. legen sich die auf der Aussenfläche der Helix und vor derselben stehenden Haare. Bei einem Tauchversuch wurde beobachtet, dass sich der zwischen Helix und Crus anthel. sup. liegende Scaphaabschnitt in eine ausgiebige Längsfalte, die aus der Helixkammer herauskommend bis an 
den freien Scapharand lief, legte. Ähnliches wurde beim Aguti, Hydrochoerus und Opussum gefunden.

2. Triangulariskammer (Fig. 12a). Die beiden Crura rücken gegeneinander. Das lappenförmige Crus anthel. inf. bildet in querer Richtung eine Falte, deren Convexität gregen das Crus anthel sup. gerichtet ist, und legt sich mit dem mittleren Abschnitt seines freien Randes gegen das Crus anthel. sup. Gegen das aborale Ende des Crus anthel. inf. legt sich die rom Crus anthel. sup. herkommende Anthelixstammfalte und schliesst die Fossa triang. hier nach hinten ab. In die auf solche Weise stark verengerte und fast verschlossene Fossa triang. führt also jetzt nur am oralen und aboralen Ende des Crus inf. eine

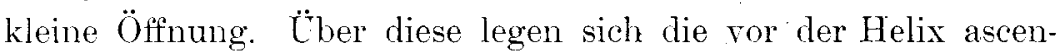
dens stehenden Haare, die bis an die kurzen ihnen entgegen gerichteten Haare der Anthelixstammfalte reichen. Am Boden der Fossa triang. verläuft vom aboralen Ende des Crus anthel. sup. her eine schräge Falte nach dem oralen Ende des Crus anthel, inf. hin.

3. Cymbakammer (Fig. 12a). Diese Kammer kann sich bei der Kontraktion der Muskeln bei weitem am stärksten verkleinern. Fig. 12a zeigt ein Vorstadium dieses Zustandes, von dem in seiner maximalen Ausbildung leider keine Aufnahme gelang. Tragus und Antitragus nähern sich einander bis zur Berührung, oder es bleibt eine schmale Lücke zwischen beiden, die die Haare des Antitragus ausfüllen. Dabei nähert sich die Plica antitragica dem Crus anthel. inf., sodass nur ein schmaler Eingang in die Cymba bleibt. Dieser Eingang ist ungefähr dreiseitig. Er wird begrenzt von der Helixwurzel, dem oberen Rand des Tragus und dem Antitragus, dem Crus anthel. inf.

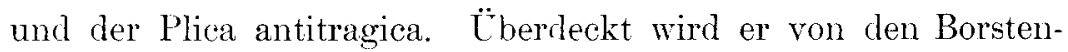
haaren des Antitragus, der Plica ant. und den spärlichen jenen entgegen gerichteten Haaren des Crus anthel, inf. Über alle dicse hinweg legen sich die vor dem Ohr stehenden Haare 
Durch den geschilderten Eingang blickt man in die Cymbakammer. Doch erstreckt sich diese noch ein Stück weiter kaudal, wo ihre Wände vom Boden der Cymba, der Anthelixstammfalte und dem Antitraguswulst gebildet werden. Es kann aber auch die Verkleinerung der Cymbakammer weniger stark sein. Dann bleibt der Fingang weiter. Seine Form ist in diesem Falle länglich vierseitig. Begrenzt wird er von der Helixwurzel, dem Crus anthel. inf., der Anthelixstammfalte, der Plica antitragica, dem Antitragus und dem sich an den letzteren anschmiegenden Tragus. - Bei manchen Tieren bildet sich beim Ohrschluss ein Wulst.(Fig. 12b), der am Boden der Concha rom kaudalen Ende des Crus anthel. sup. nach dem Porus hinläuft. Gegen diesen Wulst legt sich der Antitraguswulst. Auch sonst findet man bei den einzelnen Individuen kleine Abweichungen von dem beschriebenen Modus.

4. Cavumkammer und Porusschluss (Fig. 12b). Der auch in der Ruhe nach innen wulstförmig vorspringende Antitragus tritt jetzt in Gestalt eines starken Wulstes nach inmen hervor and legt sich gerade auf den Porus acust. Dieser wird dadurch fast vollständig rerschlossen. Nur oralwärts von dem Antitragus bleibt eine minimale Öffnung. Die Incisura intertrag. schliesst sich. Der Schluss der Incisura intertrag. ist, wie gesagt, njeht immer vollständig. Es kann an ihrem ventralen gerundeten Ende eine Öffnung bestehen bleiben. Diese führt in einen Raum (Cavumraum), der vom Tragus, dem Antitraguswulst, dem Borlen des Cavum begrenzt wird.

Die dünne Hautfalte am Porus ac. ext. tritt scharf hervor. Es verzieht sich auch dieselbe. sodass der Eingang schräg dorsalwärts in die Länge gezogen wird, wobei er sich verschmälert. Er schliesst sich aber nicht etwa durch ringförmige Kontraktion, sondern wird durch Auflagerwng des Antitraguswulstes geschlossen.

Nur die Bildung der Cymba- und Carumkammer und der 
Verschluss des Porus tragen zu einem Abschluss des äusseren Gehörganges bei. $\mathrm{Ob}$ die Bildung der Helix- und Triangulariskammer eine Bedeutung hat, vielleicht die, das Eindringen ron Erde etc. zu verhindern, oder ob sie einen Atavismus vorstellt oder nur eine durch gleichzeitige Innervierung der betreffenden Muskeln hervorgerufene zwecklose Begleiterscheinung ist, vermag ich nicht zu sagen.

Sobald man das Tier ins Wasser setzt, beginnt es zu schwimmen. Die Ohrmuscheln werden sofort nach hinten und dem Kopfe an gelegt. Die Helixkammer bildet sich in vorzüglicher Weise. Ihre Innenwandung bleibt trocken, auch wenn das Tier mehrere Male untergetaucht wurde. Ebenso tritt die Bildung der Triangulariskammer gleich stark ein wie bei elektrischer Reizung. Nicht so dicht wie bei letztgenanntem Reiz ist der Abschluss der Cymba. Dagegen schliesst sich die Incisura intertragica ziemlich eng. Die Prüfung mit Kalium hypermanganicum (s. u.) ergab denn auch, dass das Wasser bis in die Cavumkammer gelangt. Nimmt man das Tier aus dem Wasser heraus und setzt es hin, so schüttelt es heftig den Kopf, um das in die Ohren eingedrungene Wasser herauszuschleudern. Nur wemn es erschöpft ist. was auch nach kurzdauerndem Schwimmen oft der Fall ist, braucht es erst längere Zeit, um sich zu erholen und' verzichtet auf die Ohrreinigung. Das Meerschweinchen hat also die Fähigkeit, die Ohrmuschel zu schliessen, doch genügt der Schluss meist nicht, um das Eindringen des Wassers zu verhüten. Es macht das Gebaren des Tieres im Wasser auch den Eindruck, als ob der Aufenthalt in diesem Element ihm durchaus fremdartig sei. Hiermit steht vielleicht jene mangelhafte Funktion im Zusammenhang. Danach hat beim Meerschweinchen der Ohrverschluss wohl den Zweck, das Eindringen von Erde und Sand zu verhüten. 
Aguti (Dassprocta aguti).

Die Ohrmuschel des Aguti ähnelt der des Meerschweinchens. Von den im einzelnen bestehenden Abweichungen sei ein warzenähnliches Gebilde ror dem Tragus und ein ähnliches in der Cymba am kranialen Anfang des Tragus erwähnt. Bei Reizung mit dem Induktionsstrom tritt ein vorzüglicher Incisurschluss ein. Dabei nähern sich die genannten warzenähnlichen Höcker der Incisura. Es erfolgt eine ziemlich bedeutende Verengerung des Conchaeingangs durch Wirkung dei Anthelixstammfalte. Auf der Scapha bildet sich eine Längsfalte. Endlich beobachtet man wie beim Meerschweinchen eine Helixkammer.

Die Beobachtungen, die in den Zoologischen Gärten ${ }^{1 j}$ an lebenden Tieren gemacht wurden, seien in folgendem kurz wiedergegeben. Ebenso sei ron meinen Untersuchungen an konserviertem Material, die ich im naturwissenschaftlichen Museum zu Berlin und Hamburg ${ }^{1}$ ) anstellte, dasjenige angeführt, was für die uns hier interessierende Frage in Betracht kommt. Der Ühersichtlichkeit wegen sind die Beobachtangen an lebendem und totem (Spiritus-) Material nicht getrennt, sondern nebeneinander bei den einzelnen Familien angeführt.

Pitheci. (atarrhini. Simia troglodytes (Zoo. Mus. Berl.). Die Ohrmuscheln variieren in Form und Grösse stark, wie auch eine Kollektion von Photographieen. die mir Herr Prof. Matschie zu zeigen die Freundlichkeit hatte, erkennen liess. Ein Exemplar des Zoo. Mus. Berl. hat ausserordentlich grosse Ohren. was natürlich auf der Aushildung der Scapha beruht. Letztere zeigt nur eine kurze Helix. Der Stamm der Anthelix ist viel weicher als beim Menschen und macht vielmehr deu Eindruck einer ausgleichbaren Falte. Er läuft direkt in das Crus anthel. sup. aus, während das Crus anth. inf. eine isolierte, stark ausgebildete Horizontalfalte ist. Danach hat man den Eindruck, dass die Anthelix im Ganzen rom Anthelixstamm und dem Crus anth. sup. gebildet wird und dass das Crus

1) Den Herren Brauer. Matschie, Heck. Heinroth (Berlin), Bolau. Kräpelin, v. Brunn, Hagenbeck, Sokolowski (Hamburg), Priemel (Frankfurt a. M.) bin ich für die Erlaubnis, an den genannten Orten Untersuchungen anstellen zu dürfen, zu Dank verpflichtet. 
anth. inf. ein selbständiges Gebilde ist. - Anthropopithecus calvus (UnterGuinea), (Zoo. Gart. Berl.), Ohren gross, unbeweglich. - Pithecus satyrus (Zoo. Gart. Frankf.), Ohren unbeweglich - Hylobates spec. (Zoo. Mus. Berl.). Gut. ausgebildete Helix. Die Concha ist breit. Das Crus anthel. inf. sieht ans wie die Fortsetzung des Anthelixstammes, während das Crus sup. sehr schwach ist. Das Crus helicis zieht in die Concha hinein, aber nicht sehr tief. Die Incisura intertragica ist weit. In der Mitte des Scapharandes findet sich die Andeutung einer Plica longitud. (?). Bei einem anderen Exemplar ist dagegen das Crus anth. sup. deutlich und stellt die direkte Fortsetzung des Stammes vor. Das Crus inf. liegt in einem tieferen Niveau. - Hylobates leuciscus (Zoo. Mus. Hamburg hat ein nur schwach angedeutetes Crus anthel. infer. - Semnopithecus (Zoo. Yus. Berl.). Stamm der Anthelix und Crus sup. stellen ein einheitliches Gebilde vor. Auch die Art der Behaarung zeigt, dass das Crus. sup. die direkte Fortsetzung des Stammes ist. Beide liegen in einem höheren Niveau als das Crus inf. Die Helixwurzel zieht weit in die Concha hinein. Auf der Scapha Andeutungen von 2 Plicae longit. (?). Bei einem anderen Exemplar, S. entellus, zeigen die Crura dieselben Beziehungen zum Stamm, bei einem dritten dagegen bildet das Crus inf. ein Einheitliches mit dem Anthelixstamm. - S. flavimanus (Zoo. Gart. Berl.) Ohren ganz unbeweglich. -Colobus vellerosus (Zoo. Mus. Berl.). - Gut ansgebildete Helix mit weit die Concha durchquerendem Crus. Anthel. mit zwei Crura; das inf. ist scharf begrenzt und setzt die Richtung des Stammes fort. - Cercopithecus aethiops (Zoo. Gart. Berl.), Ohr unbeweglich. Cercopithecus spec. (Zoo. Mus. Berl.). Das Crus anth. sup. bildet die Fortsetzung des Stammes, das inf. ist scharfkantig. mehr selbständig. - Macacus (Inuus) crnomolgus (Zoo. Mus. Berl.). Das Crus anth. sup. setzt direkt den Stamm der Anthelix fort und zwar nicht nur in der Richtung, sondern auch in der Form als breite abgerundete Falte, während das Crus inf. selbständig und scharfkantig ist. - M. leoninus (Zoo. Gart. Berl.). Ohr ganz unbeweglich. - M. fuscoater (Zoo. Gart. Berl.) zeigt eine Andeutung von Anthelixschluss. - M. rhesus (Institut Giessen). Bei elektrischer Reizung tritt eine Verengerung der Incis. intertrag. ein und deutliche Bewegung der Anthelix. - Pitheci. Platyrrhini. Ouakaria (Zoo. Mus. Berl.). Die Anthelix stellt eine scharfe unterminierte Ringfalte vor. Da ein, wemm auch nur schwach ausgebildetes Crus anth. sup. vorhanden ist, so wird das kraniale Ende der Anthelix von dem Crus anth. inf. gebildet, das also hier ein Einheitliches mit dem Stamm bildet, wie so oft beim Menschen. - Lagothrix lagothrica (Zoo. Mus. Berl.). Die Crista anthel, anterior überragt im Niveau ziemlich weit die Helix. Dadurch ticfe Fossa scaphoidea. - Ateles Geoffroyi 
(Zoo. Gart. Frankf.). Ohr unbeweglich. (Zoo. Gart. Berl.). Deutlicher, wenn auch geringer Anthelixsehluss. - A. beelzebuth (Zoo. Wus. Berl.). Kein deutliches Crus anth. sup., wohl aber inf., das die Richtung des Stammes fortsetzt. Gut ausgebildete Helix mit nach innen umgekrempter Scaphaspitze. - Cebus spec. (Zoo. Mus. Berl.). Ohr ähnlich wie bei Macacus, auch das Verhalten der Anthelix ebenso. Dieselbe Art der Behaarung geht vom Stamm auf das Crus sup. iber. - Pithecia leucocephala (Zoo. Mus. Berl.). Crus anthel. sup. schwach, Crus inf. kräftig ausgebildet. Grosser Antitrag., kleiner Tragus.

Callithrix moloch. (Zoo. Gart. Berl.). Ohr ganz unbeweglich. - Zusammenfassung: Bei den Affen konnte die Fähigkeit, die Ohrmuschel zu schliessen nicht nachgewiesen werden. Andeutungen von einem Ohrschluss wurden beobachtet bei Macacus fuscoater. Macacus rhesus, Ateles Geoffroyi. Bei vielen Affen besteht die Anthelix wie beim Menschen aus einem Stamm und zwei Crura. Das Crus sup. steht in engerer Beziehung zum Stamm als das Crus inf. Nach unserer Auffassung stellen diese beiden Teile zusammen eine Halbringfalte, die Schlussfalte der ehemaligen schlussfähigen Form, auf die auch das Affenohr (siehe unten) zurückzuführen ist, vor. Die Tatsache, dass das Crus sup. oft in der Richtung des Stammes wie bei vielen menschlichen Ohren - verläuft, kann nicht als Beweis für unsere Annahme dienen. da dies auch oft für das Crus inf. gilt z. B. bei dem einen Hylobates, einen Semnopithecus, Colobus vellerosus, Ouakaria, Ateles beelzebuth, Pithecia leucocephala. Dagegen spricht für jene Aunahme, dass das Crus inf. bei manchen Affen eine isolierte, oft in einem tieferen Niveau liegende, oft longitudinal verlaufende Falte ist. Das Crus sup. steht dagegen, wenn es vorhanden ist, stets im $\mathrm{Zu}$ sammenhang mit dem Stamm und liegt in demselben Niveau wie dieser: weiter spricht dafür, dass das Crus sup. stets dieselbe abgerundete, breite Wölbung zeigt wie der Stamm. das Crus inf. aber meist scharfkantig ist. endlich, dass sich bei einigen Affen die für den Anthelixstamm charakteristische Bchaarung direkt auf das Crus sup., nicht aber auf das Crus inf. fortsetzt. - Dass die Gestalt der Ohrmuschel variiert, wurde beobachtet bei Simia. Hylobates, Semnopithecus.

Prosimii. Lemur macaco (Zoo. Mus. Hamburg) hat nur eine kurze Crista anth. ant., die nach ihrer (ventralen) Lage ein Crus inf. vorstellt. Dafür spricht auch, dass sich darüber eine Grube findet, die der Fossa triang. entsprechen wiirde. Ein Anthelixstamm und Crus anth. sup. fehlen (Zoo. Gart. Berl.). Ohr unbeweglich. - Lemur catta (Zoo. Gart. Berl.). Ohr unbeweglich. -- Lemur varius (Zoo. Mus. Hamburg) zeigt ein lappenförmiges Gebilde, von dem es fraglich ist, ob es ein Crus sup. oder inf. ist. - Lepilemur 
(Zoo. Mus. Berl.). Kein Anthelixstamm vorhanden, wohl aber eine Plica antitragica und ein Crus anth. inf. Dass diese stark vorspringende scharfe Falte das Crus inf. ist, geht aus seiner Lage hervor. Ventral von ihm könnte kein Crus weiter liegen. - Nycticebus tardigradus (Zoo. Mus. Hamburg). Das Crus anth. inf. bildet einen grossen Lappen, das Crus sup. eine Umbiegungsfalte. - Stenops gracilis (Zoo. Mus. Berl.). Die Ohrmuschel zeigt zwei grosse lappenförmige Gebilde, die man nach ihrer Lage als Crura anthelicis anzusehen geneigt ist. Die Plica antitragica ist ebenfalls lappenartig gestaltet. Sie bildet mit der Scapha eine Tasche. - Galago ist bekanntlich imstande, die ganze Scapha einzurollen. - Galago spec. u. G. Kirki (Zoo. Mus. Berl.) besitzen eine kulissenartig vortretende C'rista anth. ant. -. Otolicnus apicalis (Zoo. Mus. Berl.) zeigt eine ihrer Lage nach als Crus anth. inf. zu bezeichnende leistenartige Falte. Ein Anthelixstamm und Crus sup. fehlen. -- Otogale lasiotis (Zoo. Mus. Berl.). An der Basis der Scapha am kranialen und kaudalen Rande je eine taschenartige Bildung. - Tarsius spectrum (Zoo. Mus. Berl.।. Die Crista anth. ant., Tragus und Antitragus sind lappenartig. - Galeopithecus (Berl. Zoo. Mus.). Ohrmuschel ausserordentlich einfach. Die Anthelix ist nur als Umbiegungsfalte angedeutet, tritt nirgends als Leiste herror. Zusammenfassung: Bei den Prosimiern ist meist eine einfache Crista anthel. anterior von lappenförmiger Gestalt vorhanden, die wir ihrer Lage nach für ein Homologon des Crus. anth. inf. des menschlichen Ohres halten. Diese Auffassung wird dadurch gestützt, dass sich bei Nycticebus ausser einem lappenförmigen Gebilde, das der Lage nach der Crista anthel. ant. der ubrigen Prosimier entspricht, ein kranial davon liegendes Crus in Gestalt einer Umbjegungsfalte findet. Demnach stellt das erstere ein Crus anth. inf., das letztere das Crus anth. sup. vor. - Andeutungen von einer Schliessfähigkeit der Ohrmuschel konnte bei Prosimiern nicht nachgewiesen werden. Eine Ausnahmestellung nehmen die Galagos ein, die bekanntlich, wenn sie schlafen wollen, ihre grossen Ohrmuscheln einrollen und auf solche Weise đen Eingang zum Gehörgang verschliessen. - Wie man sielnt haben uns unsere Untersuchungen zu derselben Anschauung geführt wie sie Schwalbe bereits früher (1s89, 1. c. p. 249) ausgesprochen hat, dass nämlich die Crista anth. ant. dem Crus. anth. inf. entspricht. Auch die verschiedene Form der beiden Crura und das Getrenntbleiben des Crus inf. vom Stamm der Anthelix beim Menschen hebt Schwalbe an genanntem Orte hervor. Im Folgenden werden wir bei tiefer stehenden Familien Gebilde, die in Form und Lokalisierung der Crista anthel. ant. entsprechen als solche bezeichnen, ohne aber damit schon jetzt Bestimmtes über ihre Homologie sagen zu wollen. 
Chiroptera. Pteropus (Zoo. Mus. Berl.). Ohrmuschel sehr einfach. Scapha gross. Ein Crus anth. inf. (?) angedeutet. - Pterocyon stramineus (Zoo. Gart. Berl.). Die grosse löffelförmige Ohrmuschel als Ganzes sehr beweglich. - Die Ohren der Unterordnung Insectivora sind z. T. kompliziert gestaltet und eigenartig in ihrer Bewegung. Sie rerlangen ein eigenes studium. Erwähnt seien die ausserordentlich grossen Ohrmuscheln von Plecotus auritus. Das Tier legt. wie wir anch an unserem gefangenen Exemplar beobachten konnten, beim Schlafen und auf Anblasen die Ohren nach hinten und schliesst die Scapha.

Carnivora. Felis domestica (Giessen). Bei elektrischer Reizung tritt ein vorzüglicher Abschluss des Carums ein. - Felis melas (Zoo. Mus. Berl.). Das jugendliche Exemplar zeigt keine vollständige Anthelix, sondern nur eine Crista anth. ant. Diese hat die Gestalt einer halbkreisförmigen, ziemlich dicken (fein behaarten! Scheibe. Dort, wo sich das kaudale Ende des Anthelixstammes finden würde, sieht man warzenförmige Gebilde, von denen eins fast schalenfömig ist. Letzteres ist jedoch nicht etwa der Antitragus mit der Plica antitrag. Dazu liegt es zu weit ohrinnenwürts und anch nicht gegenüber vom Tragus. Das Cavum conchae ist sehr geräumig. Die Hauttasche an der Scapha ist gut ausgebildet, innen gleichmäligig behaart; die imnere Hautplatte dex Tasche ist ausgezackt. Man gewinnt den Eindruck, dass jene innere Platte dem Antitragus genauer der Plica antitrag. entspricht. -.. (ynailurus Soemmerringii (Zoo. Gart. Frankf.) legt die Ohrmuschel nach hinten und schliesst die Scapha. - Hyaena crocuta (Zoo. Gart. Frankf.). Auf mechanische Reize wird die Ohrmuschel nach hinten gelegt; die Scapha wird längs gekielt, indem sich ein sehmalerer oraler Teil anf den breiteren kaudalen legt. - Hyaena striata (Zoo. Lus. Berl.). Ganz junges Tier. Aus der Concha treten pilzförmige Gebilde - wohl zur Anthelix gehörig - hervor. Fie relschliessen den Zugang zum Porus vollständig. -- Canis familiaris (Giessen). Auf elektrische Reizung erfolgt Verengerung der Incisur. Der Antitragus nähert sich dem Conchaboden, die laterale Cavumwand dem Porus. - Canis variegatus (Zoo. Mus. Berl.). Embryo von $16 \mathrm{~cm}$ Scheitelsteisslänge über den Rücken gemessen. Der Conchaeingang ist vollständig geschlossen. Hier finden sich in der Gegend des Anthelixstammes Verdickungen, die mit schwach angedeuteten Plicae longit. in Verbindung stehen. Dieses Entwickelungsstarlium lässt die Entstehung der Hauttasche der Scapha erkennen. Danach wird die innere Platte vom Antitragus und der Plica antitrag. und dem proximalen Teil der kaudalen Plica long. gebildet. Die beiden ersteren haben hier noch die Stellung, wie sie bei vielen Ohrformen, z. B. auch beim menschlichen 
während des ganzen Lebens erhalten bleibt. Später klappen sie sich nach unten um und bilden die innere Platte der Hauttasche. Die Plica antitrag. geht distalwärts in die kaudale Plica long. uber. Wo dies geschieht, ist ein Finschnitt. Es ist dies derselbe Einschnitt, der sich bei erwachsenen Carnivoren so häufig an dem inneren Blatt der Hauttasche findet. Seine Entstehung und sein frühzeitiges Auftreten macht seine Konstanz verständlich. Die äussere Platte ist ein Teil der Scapha. - Viverra genetta (Zoo. Mus. Berl.). Der orale Rand der grossen Scapha läuft nach vorn und medialwärts auf den Kopf aus. Ein dickes lappenförmiges Gebilde scheint das Crus anthel. inf. vorzustellen. Ein Anthelixstamm ist nicht vorhanden. Dort wo sein kaudales Ende liegen würde, liegen zwei Verdickungen, die sich verjüngend anf die Scapha auslaufen und so den Eindruck machen, als wären sie die proximalen Enden verschwundener Plicae longitudinales. Das ganze Ohrinnere ist unbehaart. Hauttasche an der Basis des hinteren Scapharandes. Die innere Platte der Hauttasche zeigt einen grossen winkelförmigen Ausschnitt. - Herpestes zebra (Zoo. Mus. Berl.). Die Scapha ist im Verhältnis zur Concha nur klein. Ejn eigentlicher Stamm der Anthelix ist nicht vorhanden, sondern nur eine Umbiegungsfalte als Grenze zwischen Concha und Scapha. Die Crura anth. sind zwei lappenförmige Gebilde, von denen das Crus inf. das grössere ist. Die zwischen ihnen gelegene Fossa triang. ist lang und schmal. - Herpestes griseus (Zoo. Gart. Frankf.) vermag auf Kitzeln mittels der grossen lappenförmigen Crura anth. den Conchaeingang vollständig zu schliessen. Vielleicht macht er hiervon Gebrauch, um das Eindringen von Sand. etc. zu verhüten, wenn er euge Höhlen und Gänge nach Beute, z. B. Schlangen, durehforscht. - Bdeogale crassicauda (Zeo. Mus. Berl.). Die Ohrmuschel ist ähnlich gestaltet wie bei Herpestes nur ist die Scapha grösser. - Galidia elegans (Zoo. Gart. Berl.). Ein Anthelixschluss tritt auf Anblasen und Kitzeln nicht ein. - Rhyzaena (Zoo. Mus. Berl.). Scapha klein. An Stelle des Anthelixstammes eine Umbiegungsfalte. Gut ausgebildetes Crus anth. inf. Scapha sowie das ganze Ohrmuschelinnere behaart. --. Helogale undulata (Zoo. Mus. Berl.). Scapha klein; Crus anth. sup. klein: Crus. anth. inf. grosses dickes kulissenartiges Gebilde. -- Mustela zibellina (Zoo. Gart. Berl.). Scaphainnenfläche reichlich behaart. - Putorius vulgaris (Zoo. Mus. Berl.) wie bei P. ermineus. - Putorius foetidus und P. boccamela (Zoo. Mus. Berl.) wie bei P. ermineus, doch scheinen die Knoten im kaudalen Ende der Anthelixstammfalte weniger stark ausgebildet zu sein.-Putorius sarmaticus (Zoo. Mus. Berl.) wie bei P. foetidus, doch fehlten bei diesem Exemplar die Haare auf den knotenförmigen Verdickungen. - Galictis 
barbara (Zoo. Gart. Berl.). Ganz kurze, auf der Innenfläche behaarte Scapha. Auf mechanischen Reiz tritt ein sehr guter Anthelix- (Concha-)schluss ein. Lutra (Zoo. Mus. Berl.). Die Ohrmuschel ist auch auf ihrer Innenfläche dicht behaart, sodass sich ihre Form unter der Behaarung verbirgt. Die Crista anthel. ant. ist kugelig, reichlich mit langen Haaren bestanden. Am kaudalen Ende der Anthelixstammfalte kleine knollige Verdickungen. Der Porus ac. liegt im Carum conchae, das sich weit ventralwärts erstreckt. - Mellivora ratel (Zoo. Gart. Berl.). Eine aus dem Niveau der Umgebung sich hervorhebende Ohrmuschel fehlt. Es macht durchaus den Eindruck, als ob das Tier den schlitzförmigen Ohreingang schliessen könnte. - Rabdogale (Zoo. Mus. Berl.j. Die Crista anthel, ant. stellt einen verdickten, gleichmäfig diun behaarten Lappen ohne knollige Anschwellung vor. - Cercoleptes caudivolvulus (Zoo. Gart. Frankf.) legt auf Kitzeln die Ohrmuschel zurück, wobei sich der schmalere orale Längsabschnitt der Scapha auf den breiteren aboralen auflegt. Ein Anthelixschluss trat jedoch auch auf Reizung mit dem Induktionsstrom nicht ein. - Procyon cancrivorus (Zoo. Mus. Berl.). Crista anth. ant. knollig. An der Anthelixstammfalte kranial und kaudal eine knollige Verdickung. - Ursus maritimus (Zoo. Gart. Frankf.). Ohrmuschel sehr klein, innen stark behaart. - Zusammen fassung: Ein Scaphaschluss kommt bei den Carnivoren häufig ror. Ein Verschluss durch lappenartige zur Anthelix gehörige Gebilde wurde bei Herpestes, ein sehr vollkommener Conchaschluss mittels der Anthelix bei Galictis beobachtet. - Knollige Verdickungen im kranialen und kaudalen Abschnitt der Anthelix finden sich bei Vertretern fast aller Familien der Carnivoren. - Bei den verschiedensten Familien der Carnivoren findet sich am aboralen Kande der Scapha eine Hauttasche. - Auf der Innenfläche behaarte Ohrmuscheln finden sich bei Carnivoren, die sich viel im Wasser aufbalten.

Insectivora. Erinaceus europaeus siehe oben. - Centetes ecaudatus (Zoo. Mus. Hamburg). Am hinteren Rand der Scapha Andentung einer Tasche. - Macroscelides rozeti (Zoo. Gart. Frankf.). Crista anth. ant. lappenförmig. Das Tier vermag nach mündlicher Angabe von Dr. Priemel die Ohrmuschel zu schliessen. - Petrodromus (Zoo. Mus. Berl.). Crista anth. ant. kulissenartig vorspringend. - Crocidura und Sorex s o. - Crocidura caerulescens (Zoo. Mus. Berl.) hat wie unsere einheimische zwei Schalen in der Ohrmuschel die an ihren freien Rändern mit ziemlich langen Haaren besetzt sind. Potamogale. Die Incisura tragohelic. bildet eine Rinne. Am Antitragus eine Reihe Haare, die vielleicht eine Rolle bei der Verhinderung des Eindringens von Wasser spielen. - Talpa ist bekanntlich imstande, den Eingang zum 
Gehörgang zu schliessen. - Zusammenfassung: Bei verschiedenen Arten der Insektivoren finden sich verschiedene Modi von Ohrschluss.

Rodentia. Sciurus vulgaris scheint die Ohrmuschel schliessen zu können. - Sciurus bicolor (Zoo. Mus. Berl.). Der Antitragus verhält sich zur Scapha wie die Helix, stellt also den eingekrempten aboralen Rand der Scapha. vor. - Heliosciurus rufobrachiatus (Zoo. Mus. Berl.). Die Anthelix ist eine Umbiegungsfalte. Mit Hilfe dieser, sowie des Tragus und des deckelartigen Antitragus kann man am Spirituspräparat die Concha vollständig schliessen. - Sciuropterus volans (Zoo. Mus. Berl.). Die Concha besitzt eine kaudal gerichtete Ausbuchtung. - Xerus capensis (Zoo. Gart. Berl.). Die Scapha ist sehr klein. Sehr guter Conchaschluss. - Aretomys marmota (Zoo. (iart. Berl.). Scaphainnenfläche behaart. Guter Conchaschluss. -- Muscardinus s. o. - Castor fiber (Zoo. Gart. Berl.). Ohrmuschel auch an der Innenfläche behaart. Durch eine Längskielung teilt sich die Scapha in einen schmalen dorsalen und einen breiten ventralen Abschnitt. Sobald das Tier tauchen will, legt es mit einer sichtharen Bewegung den ersteren fester gegen den letzteren. Nach dem Emportauchen ist keine Bewegung sichtbar. Vielleicht geschieht das Nachlassen der Muskelkontraktion so allmählich, dass man die Bewegung nicht sieht. Der Mechanismus, wodurch das Eindringen des Wassers verhindert wird, muss sehr vollkommen funktionieren, da das Tier nach dem Auftauchen auch keine Schleuderbewegung mit dem Kopfe macht Beim Jungen (Zoo. Mus. Berl.) ist die Behaarung auf der Innenfläche des Ohres samtartig dicht. -- Thomomys cinnamomeus (Zoo. Mus. Berl.). Ohrmuschel wie eine kurze Röhre gestaltet. -- Pedetes caffer (Zoo. Gart. Berl.). Grosse Scapha, langer, zylindrischer Tragus, einfache aber vollständige Anthelix. Legt auf Anblasen die Ohrmuschel zurück, wahrscheinlich wird dabei auch die Anthelix gegen den Conchaboden gedrückt. Perognathus fasciatus (Zoo. Mus. Berl.). Die Anthelixfalte ist ziemlich tief unterminiert, der Antitragus ausserordentlich gross. - Dipus hirtipes (Zoo. Gart. Frankf.). Vollständiger Scapha- und Anthelixschluss. Dipus aegyptius Von dieser Spezies sagt Brehm: Die Ohren werden beim Schlafen dicht an den Kopf gedrückt und ihre Spitze teilweise eingerollt, sodass sie faltig, gleichsam wie zerknittert aussehen. - Tatera hirtipes s. o, - Zapus hudsonius (Zoo. Gart. Berl.). Die grosse Plica antitragica legt sich gegen den Conchaboden und schliesst den Eingang in das Cavum cymbae gut ab. - Cricetus frumentarius s. o. - Mus sylvaticus, M. musculus, M. agrarius s. o. - Mus minutus (Giessen). Beim Schlussmechanismus tritt der kraniale Abschnitt der Anthelixfalte stark hervor. Die Luft fängt sich beim Tauchen hinter der grossen 
Plica antitragica und verhindert das Eindringen des Wassers in das Cavum. - Mus decumanus s. o. - Wus rattus (Zoo. Mus. Berl.). Scapha bedeutend grösser als bei M. decumanus. Die ganze Ohrmuschel ist weicher und macht einen schlafferen Eindruck als bei decumanus. Die Incisura intertrag. ist bei letzterer enger. M. rattus zeigt also in Bezug auf den Schlussmechanismus eine weniger gute Anpassung an das Wasserleben als decumanus. - Mus alexandrinus (Zoo. Mns. Berl.) wie M. rattus. - Meriones spec. (Zoo. Mus. Berl.). Die Anthelix ist leistenartig ausgebildet. Auch bei diesem Tier liegt der Porus ac. in der Tiefe des Cavums. - Dendromys spec. (Zool. Mus. Berl.). Riesige Plica antitragica, die sich deckelartig über den Tragus herüberlegt. Kulissenartig hervortretende Crista anthel. ant. - Hydromys beccari (Zoo. Vus. Berl.). Der Tragus setzt sich nicht gegen die Helix ascendens ab. Der Porus liegt wie bei vieleu Tieren weiter ventralwärts als das geschlossene Ende der Incisura intertrag. - Hypudaeus glareolus s. o. - Arvicola arvalis s. o. - Arvicola amphibius :Zoo. Mus. Berl.). Grosse plattenartige Plica antitragica. -- Myodes torquatus (Zoo. Mus. Berl.). Ohrmuschel sehr klein, weit offene Concha, sehr kleine Scapha mit kurzer Helix, keine Incisura intertrag. - Georhychus Mechowii (Zoo. Mus. Berl.). Ohr ohne hervortretende Ohrmuschel, wie bei Talpa, dem das Tier in der Lebensweise ahnelt. Den Ohreingang umgibt ein Wulst: -- Cavia aperea (Zoo. Mus. Hamburg). Ohrmuschel wie bei C. cobaya. - Cavia cobaya s. o. - Dasyprocta aguti s. o. - Hydrochoerus capybara (Zoo. Gart. Hamburg). Ohrmuschel ähnlich wie bei Cavia. In der Scapha kann eine Längsfalte auftreten. Aulacodus Swinderenianus (Zoo. Mus. Berl.). Ohrmuschel ähnelt der von Cavia; zeigt ein grosses lappenförmiges Crus anth. sup. und ein ähnliches kleineres Crus anth. inf. Der freie Rand des ersteren ist mit Haaren besetzt. Zwischen beiden eine grosse Fossa triangularis. Die Innenfläche der Scapha ist behaart. -Capromys Fournieri (Zoo. Mus. Berl.). Das Spirituspräparat macht es sehr wahrscheinlich, dass ein Anthelix- und Incisuraschluss möglich ist. Leistenartiges Crus anth. inf. - Myopotamus coypus (Zoo. Gart. Berl.). Ohrinnenfläche sehr lang behaart. Guter Conchaschluss, dabei bildet sich in der Scapha eine längsfalte wie bei Cavia, aber stärker. Nach dem Tauchen ist die Behaarung der Concha absolut trocken. - Lagostomus trichodactylus (Zoo. Gart. Frankf.). Im schlaf wird die Scapha nach hinten gelegt. Wahrscheinlich tritt auch eine Verengerung des Conchaeinganges ein. - Zusammenfassung: Bej den Rodentia scheint die Anthelix in den meisten Fällen einfach zu sein. Lappenartige Gebilde, die wohl den Crura anthelicis entsprechen, finden sich hei den Subungulaten und bei Aulacodus. Das Vermögen, die Ohrmuschel 
zu schliessen ist bei den Rodentia ausserordentlich häufig. Es waren sämtliche (ca. 19) geprüfte Spezies, die sieben verschiedenen Familien angehören, hierzu imstande.

La am nunguia. Hyrax capensis (Zoo. Mus. Berl.). Das I'räparat macht den Eindruck, als könnte das Ohr geschlossen werden. Die dicke, lappenförmige Anthelix, Tragus und Antitragus sind nur durch einen sternförmigen Spalt von einander getrennt, sodass sie leicht einander genähert werden können (Zoo. Gart. Frankf.). Das Tier bewegt auf Kitzeln das Ohr nicht.

A rtiodactyla. Hippopotamus amphibius (Zoo. Mus. Berl.). Der Eingang in den Muschelgrund ist infolge der wulstförmig an der Wand vorspringenden Gebilde sehr eng. (Zoo. Gart. Frankf. u. Berl.) In dem Augenblick, wo das Ohr beim Tauchen unter Wasser kommt, legt das 'Tier die Ohrmuschel etwas nach hinten. Wahrscheinlich findet dabei gleichzeitig eine Verengerung des Eingangs der Röhre statt oder wie Boenninghaus (I. c. p. 208) meint, eine Art Abknickung des Mcatus. Beim Auftauchen schleudert das Tier jedesmal durch eine ausserordentlich kräftige Schüttelbewegung der Ohrmuschel das anhaftende Wasser ab. -- Poreus babyrussa (Zoo. Gart. Frankf.) legt auf Kitzeln das Ohr nach hinten und scheint die Scapha zusammen zu legen. Bei den Artiodactyla und Perissodactyla scheint der Ohrschluss nur vereinzelt vorzukommen.

Edentata. Manis (Zoo. Nus. Hamburg). Der Porus liegt in einem flachen Felde. Eine Strecke dahinter liegt ein niedriger Wulst, der wahrscheinlich die Scapha vorstellt. - Myrmecophaga tamandua (Zoo. Mus. Berl.). Grosse Scapha. Die die Concha umgebenden Gebilde sind einander genähert und verdickt. sodass es den Anschein hat, als könnte das Ohr geschlossen werden. - Tatus novemcinctus (Zoo. Gart. Berl.). Scapha mälig gross. Der Eingang in den Ohrgrund ist eng. Es macht den- Eindruck als könnte hier ein Schluss eintreten. - Bradypus tridactylus (Zoo. Mus. Berl.). Seapha sehr klein. Das Crus helicis erstreckt sich die Concha durchquerend bis an die Scapha. - Choloepus Hoffmanni (Zoo. Mus. Berl.). Das Carum conchae ist eng spaltförmig, sodass es leicht geschlossen werden künnte. -- Z u s a m m e nfassung: Nach der Gestalt der Ohrmnschel zu urteilen dürften verschiedene Edentaten imstande sein, die Ohrmuschel zu schliessen.

Marsupia lia. Hypsiprymnus spec. (Zoo. Mus. Berl.) zeigt zwei schalenförmige, vielleicht zur Anthelix gehörige Gebilde. - Hypsiprymnus spec. (Zoo. Mus. Berl.). In der Concha dickwulstige Gebilde, die nur wenig oralwärts verschoben brauchen zu werden, um den Olıeingang zu schliessen. -.. Phalangista murina (Zoo. Mus Berl.) besitzt am Rand der Helix ascendens 
eine gefaltete Ausbuchtung, ausserdem eine lappenförmige Crista anth. ant. - Phalangista orientalis (Zoo. Mus. Berl.). Lappenförmige Crista anth. ant. - Phalangista vulpina (Zoo. Mus. Berl.). Scapha sehr gross, dicke leistenförmige Crista anth. ant. (Zoo. Gart. Frankf.) Auf Kitzeln wird die Ohrmuschel nach hinten gelegt, die Jncisura intertr. schliesst sich, der Conchaeingang verengert sich. -- Cuscus ursinus (Zoo. Nus. Berl.). Scapha kurz mit behaarter Innenfläche; Concha nackt mit dicker lappenförmiger Crista anth. ant. - Petaurus (Belideus) breviceps (Zoo. Mus. Berl.). Grosse Scapha : ziemlich grosse, dicke, lappenförmige Crista anth. ant. - Petaurista (Zoo. Mus. Berl.) besitzt eine lappenförmige Crista anth. ant. -- Perameles dorcyana (Zoo. Mus. Berl.). Zwei lappenförmige Crura anthelicis. Zwischen Plica antitragica und Scapha eine, nach dem freien Scapharand hin offene Tasche. Perameles (Peragalea) lagotis (Zoo. Mus. Berl.) hat eine riesige Scapha wie ein Hase; am Grunde derselben zwei grosse lappenförmige Gebilde. - Didelphis azarae (Zoo. Mus. Berl.). Helix ascendens an der Basis gefaltet; lappenartige Crista anth. ant. Andeutung einer Tasche am kaudalen Scapharande. (Zool. Gart. Frankf.) legt auf Kitzeln die Scapha nach hinten und in melrere grosse Längsfalten. Dabei tritt ein vorzüglicher Concha- und Incisurschluss ein. - Didelphys crassicaudata (Zoo. MLus. Berl.). Faltung der Helix und Crista anth. ähnlich wie bei D. azarae. - Didelphys philander (Zoo. Mus. Berl.). An der Helix asc. eine läppchenförmige Bildung; komplizierte Conchabildung. -- Yikrodelphis brachyurus (Zoo. Mus. Berl.). Lappenförmige Bildung an der Helix und lappenförmige Crista anth. ant. - Phascologale (Antechinus) maculatus (Zoo. Mus. Berl.). Grosse lappenförmige Crista anth. ant.: die Helix ascendens kommt aus der Concha hervor, trägt keinen läppchenförmigen Ansatz. Zusammenfassung: Bei den Narsupialiern finden sich häufig lappenförmige Gebilde in der Concha, die als Crista anth. ant. bezeichnet wurden. Einige Male wurde ein läppchenförmiger Ansatz oder eine Faltung an der Helix ascend. beobachtet. Das Vermögen. die Ohrmuschel zu schliessen, konnte in zwei Fïllen beobachtet werden.

\section{Zusammenfassung. Bei folgenden Tieren ist ein Schliessen} der Ohrmusthel beobachtet worden:

Macacus rhesus (angedeutet) ${ }^{1}$ ), Macacus fuscoater (angedentet), Ateles Geoffroyi (angedeutet), Galago, Plecotus, Felis domestica,

1) Der Zusatz in der Klammer bezieht sich stets nur auf den direkt davor stehenden Nimen. 
Canis familiaris (Carumschluss), Cynailurus Soemmerringii (Scaphaschluss), Hyaena crocuta (Scaphaschluss), Herpestes griseus (Lappenschluss), Putorius ermineus, Galictis barbara, Lutra. Mellivora, Cercoleptes caudivolvulus (Scaphaschluss), Pinnipedia, Erinaceus europaeus, Macroscelides, Sorex, Crocidura, Talpa, Xerus, Arctomys?, Muscardinus, Castor, Zapus hudsonius, Tatera hirtipes, Dipus hirtipes, Dipus aegyptius, Pedetes caffer, Cricetus frumentarius, Mus decumanus, M. sylvaticus, M. agrarius, M. musculus, M.minutus, Hypudaeus glareolus, Arvicola arvalis, Cavia, Dasyprocta aguti, Myopotamus coypus, Hippopotamus, Porcus babyrussa (Scaphaschluss), Tatus novemcinctus, Phalangista rulpina, Didelphys azarae.

Wie man sieht, ist in fast allen geschilderten Fällen von Schliessen der Ohrmuschel immer in ähnlicher Weise rorhanden: ein Scaphaschluss, eine Verengerung des Conchaeinganges durch Zusammendrücken der Anthelix, ein Carumabschluss durch Schluss der Incisura intertragica und eine Porusverkleinerung.

Die Untersuchungen an den Tieren in den zoologischen Gärten liessen sich selbstrerständlich nur an solchen ausführen, die bis zu eiuem gewissen Grade gezähmt waren und auch an diesen nur oberfächlich, sodass ein negatives Resultat durchaus nicht als ein definitives angesehen werden darf, "wie das wohl selbstverständlich ist. Daraus erklärt sich der geringe Umfang und die Zusammensetzung jener Liste.

Es zeigte sich, dass der eben genannte Mechanismus des Ohrschlusses bei Weitem der häufigste ist. Eine abweichende Art findet sich nur bei wenigen Tieren, so bei denen, die einer äusseren Ohrmuschel entbehren, wie bei Talpa und bei Mellivora, oder bei denen sie sehr klein ist, wie bei Xerus. Ein ron dem allgemein verbreiteten Modus abweichender Ohrverschluss findet sich auch beim Seehund, den wir allerdings nicht selbst genauer beobachtet haben, von dem wir aber eine eingehende 
Schilderung von Boenuinghaus (1. c. p. 209) besitzen. Merkwürdig ist der Schlussmechanismus auch noch, wie wir angegeben haben, beim Mungo (Herpestes griseus). Hier legen sich zwei grosse lappenförmige Gebilde derartig in die Concha hinein, dass sie den Ohreingang vollständig schliessen.

Wie verbreitet die geschilderte Fähigkeit, die Ohrmuschel zu schliessen, ist, zeigt unsere Zusammenstellung. Man sieht, dass aus den meisten Ordnungen Vertreter vorhanden sind, sodass mit Sicherheit anzunehmen ist, dass die Zahl der Säugetiere, die ihre Ohren zu schliessen vermögen, eine sehr grosse ist. und dass wir in dern Abschliessen des Ohreingangs direkt eine Aufgabe der Ohrmuschel zu erblicken haben.

$\mathrm{Zu}$ welchem Zweck dies geschieht, ist in manchen Fällen ohne weiteres klar, in anderen bleibt es jedoch zweifelhaft. Bei den Säugern, die einen grossen Teil ihres Lebens im Wasser zubringen, geschieht der Ohrschluss natürlich, um das Eindringen des Wassers zu verhüten, so bei Lutra, den Pimnipediern, Castor, Myopotamus. Dies kommt auch für diejenigen in Frage, die das Wasser gerne aufsuchen, wie die Wanderratte, oder für solche, die, da sie am Boden und in der Erde leben, zuweilen mit dem Wasser in Berührung kommen, wie die Feldmaus und andere Mäusearten. Man ist immer wieder von neuem erstaunt, wie prompt bei diesen Tieren der geschilderte Mechanismus dem Wasser gegenüber funktioniert. Bei der Wanderratte, verschiedenen Mäusen und bei Myopotamus beobachteten wir, dass die Concha und selbstverständlich auch der Gehörgang nach mehrfachem Tauchen absolut trocken waren. Wie geschildert wurde und wie man an den Figuren sieht, wird der Eingang in die Concha durehaus nicht hermetisch verschlossen, und trotzdem dringt meist kein Wasser ein. Es verhindert dies die Anordnung, die Enge und Gestalt der Spalten, die Beschaffenheit ihrer Ränder, die in bestimmter Weise angeordnete Haarbekleidung einzelner Ohrteile, die fettige 
Oberfläche der Innenfläche der Ohrmuschel, und die im Gehörgang und in der Concha eingeschlossene Luft. Dass diese Momente eine Rolle spielen, konnten wir am lebenden Tier und an Wachsmodellen, die wir uns von den zusammengefalteten Ohrmuscheln anfertigten, feststellen. Wie so oft, sorgt auch hier die Natur für eine grösstmögliche Sicherheit. Es werden dem eindringenden Wasser nicht eine, sondern mehrere Barrieren entgegengesetzt, die es überwinden muss, um in den Gehörgang zu gelangen. Lässt man z. B. eine Ratte oder Vaus, ohne sie an der Ohrmuschel zu berühren, einige Male tauchen, so durchbricht das Wasser oft nicht einmal den Scaphaschluss. Hat es diesen bewältigt, so wird es in den meisten Fällen durch den Anthelixschluss rom Eindringen in die Concha abgehalten. Selten gelangt es bis in das Cavum, da es hieran durch die Verengerung der Incisura intertrag. und die Anlagerung der Plica antitrag. an den Conchaboden verhindert wird. ت̈usserst selten und nur, wenn man das Tier stark irritierte, wurde beobachtet, dass das Wasser in den Meatus eindrang. Letzteres wird dadurch verhindert, dass sich die laterale Wand des Cavums gegen den Porus drückt und die Hautfalte am dorsalen Umfange des Porus sich ausspannt. Um dies festzustellen. wurden die Tiere zum Tauchen genötigt und dabei mehr oder weniger an den Ohren insultiert. Dann wurden sie herausgenommen, und es wurde untersucht, in welchem Raume Wassertröpfchen zu finden waren. Oder abér es wurden kleine Stückchen Kalium hypermanganicum in die Concha gebracht, deren Lösung durch Rotfärbung des Wassers sofort erkennen liess, wenn Wasser eingedrungen war.

Beim Seehund handelt es sich nach der Untersuchung ron Boenninghaus um einen passiven Verschluss. Dass der Wasserdruck beim Cavumabschluss eine Rolle spielt, indem die Plica antitrag gegen den Conchaboden gedrückt wird, haben wir z. B. bei der Rötelmaus bemerkt. 
Sobald die Tiere aus dem Wasser herauskommen. schleudern sie durch eine schnelle Schüttelbewegung der Ohren oder des ganzen Kopfes das den Ohrmuscheln anhaftende Wasser ab. Sehr bequem lässt sich dies in den zoologischen Gärten bei dem Hippopotamus beobachten, das jedesmal, sobald es emportaucht, durch eine geräuschvolle Schleuderbewegung seiner Ohren diese vom Wasser befreit. Bei anderen Tieren bleibt offenbar nicht das geringste Wasser haften, so beim Bieber und dem Coypu, die beim Emportauchen es nicht nötig haben, das Wasser abzuschleudern.

Tatsächlich ist es ja auch für die Säuger, deren Hauptaufenthalt das Wasser bildet, und für diejenigen. die mehr oder weniger häufig in die Lage kommen, zu schwimmen und zu tauchen, ein Erfordernis, dass sie die Ohren schliessen können. Im anderen Falle würde das eingedrungene Wasser die Tiere am Hören verhindern und diese wären dadurch einerseits vielfach im Aufsuchen ihrer Nahrung benachteiligt, andererseits wären sie gefährdet, da sie das Herannahen ihrer Feinde nicht hören könnten Auch könnte zurückgebliebenes Wasser pathologische Affektionen hervorrufen.

Der Ohrschluss funktioniert aber dem Wasser gegenüber durchaus nicht bei allen Species, die im Stande sind, die Ohren zu schliessen, gleich gut. So ist er z. B. in dieser Hinsicht unrollkommen beim Meerschweinchen und beim Hamster. Es sind dies Tiere, die dem Wasser möglichst aus dem Wege gehen. Sie künnen zwar beide schwimmen, aber nur ungeschickt. Besonders der Hamster zeigt dadurch, dass ihn sein Haarkleid nicht im geringsten vor der Benetzung seiner Haut schützt, dass er dem Aufenthalt im Wasser nicht angepasst ist. Wie anders verhält sich da z. B. der Bieber und das Coypu, die sich beständig im Wasser tummeln, ohne dass das Wasser in ihr Wollkleid eindringt, sodass die Haut vollständig trocken bleibt! Cavia und Hamster machen eben zu anderem gleich zu nemnenden Zwecke Gebrauch ron ihrem Ohrversehluss. 
Bei anderen Säugern dient der Ohrschluss höchst wahrscheinlich gegen Eindringen von Erde und Sand, dem die Tiere als Bewohner von Höhlen und Gängen und besonders beim Graben derselben häufig ausgesetzt wären. Dies gilt z. B. für Herpestes Sorex, Crocidura, Talpa, Xerus, Arctomys, Tatera, Cricetus, Mus, Arvicola, Tatus u. a. Beim Fehlen dieser Einrichtung würde das in das Cavum und in den Gehörgang eindringende Material letzteren bald verstopfen, das Hören erschweren und zu pathologischen Veränderungen Veranlassung geben.

Sodann wird der Ohrschluss angewendet, um starke Geräusche abzuhalten. Darauf lässt wenigstens schliessen, dass manche Tiere wie Plecotus, Muscardinus, Tatera, Mus und andere ihre Ohren schliessen, wenn man Geräusche, die ihnen unangenehm sind, erzeugt, und dass sie ihre Ohren während des Schlafes bis zu einem gewissen Grade geschlossen halten. wie dies in sehr vollkommener Weise der Galago tut.

Weiter scheinen die Winterschläfer während ihres Dauerschlafes ihre Ohren geschlossen zu halten, wenigstens haben wir dies bei Plecotus, Muscardinus und Cricetus beobachtet. Ich zweifle nicht, dass dies bei den Winterschläfern allgemein geschieht. Man kann annehmen, dass, wie sich das ganze Tier zusammenroslt, so auch die Ohrmuschel zusammengelegt wird, um die freiliegende Oberfläche zu rerkleinern und die Wärmeabgabe herabzusetzen. Oder aber es legt das Tier wie beim gewöhnlichen Schlafe so auch beim Winterschlafe die Ohrmuschel zusammen. Oder endlich wird die Ohrmuschel gefaltet und im Fell verborgen, um vor der Kälte gesichert zu werden. Es kommen wohl alle diese Momente in Betracht.

Dass viele Tiere, die die Fähigkeit des Ohrschliessens besitzen, hiervon zu mehreren verschiedenen Zwecken Gebrauch machen, leuchtet ohne weiteres ein. Man vergleiche hierüber unsere Angaben bei den einzelnen Tieren. 
Bei einer Anzahl von Säugern endlich rermögen wir nicht anzugeben, welchen $Z_{\text {weck }}$ der Ohrschluss bei ihnen hat. Hier könnten wir nur durch direkte Beobachtung des Tieres in seiner natürlichen Umgebung Antwort auf unsere Frage erhalten. Dass der Ohrmuschelschluss als zwecklose Begleiterscheinung einer anderen Bewegung auftreten kann, haben wir beim Hamster erwähnt.

Die Erkenntnis, dass die Ohrmuschel vieler Tiere die Fähigkeit besitzt, sich zu schliessen, lässt uns erst die oft recht komplizierte Gestalt dieses Gebildes verstehen, die durch seine Funktion als Schallfänger nur zum Teil erklärt wird. Freilich bleibt auch so noch mancherlei unklar. Es funktioniert also die Ohrmuschel als Schalltrichter und als Schlussapparat, bei den einen Tieren in beiden Beziehungen und zwar entweder gleich vollkommen oder in einer besser als in der anderen, bei anderen Tieren nur in einer Hinsicht. Dies entspricht der ausserordentlichen Verschiedenheit der Ohrmuschelformen. Diejenigen, die in ihrer Gestalt einem Schalltrichter am nächsten kommen und so angebracht sind, dass sie nach der Richtung des Schalles bewegt werden können, werden am besten als solche funktionieren. Dies gilt z. B. von den langen löffelförmigen Ohren vieler Huftiere und mancher Nager. Das andere Extrem bilden Ohren mit kleiner Scapha und gut rerschliessbarer Concha Sie funktionieren in erster Linie als Schlussapparat.

Betrachtet man die einzelnen Teile der Ohrmuschel auf ihre Funktion hin, so zeigt sich, dass die Scapha und ihr oraler umgeschlagener Rand, die Helix, einmal Teile des Hörtrichters bilden, sodann vielfach verwendet werden, den Scaphaschluss herzustellen, bei dem sich der orale Längsabschnitt der Scapha mit der Helix auf den aboralen herüber legt. Daraus erklärt sich die Längskielung, die öfters an der Scapha beobachtet wird, ebenso wie die Querfalten, die manche Tiere auf der Scapha erzeugen können. Letztere 
dienen, wie wir an verschiedenen Beispielen gezeigt haben. dem herübergeklappten oralen Abschnitt der Scapha als Widerlager. - Vorhandensein und Gestalt der Anthelix wird verständlich aus ihrer Aufgabe, die Concha zu schliessen. Dadurch erklärt sich ihr halbringförmiger Verlauf. Auch den Sinn der verschiedenartigen Ausbildung der Anthelix vermögen wir nun zu verstehen. Bald stellt diese nämlich nichts anderes vor als eine mehr oder weniger scharfkantige oder abgerundete Lmbiegung des Ohrknorpels, wodurch eine Trennung der Concha von der Scapha angezeigt wird, bald ist sie eine Leiste oder eine kulissenartig vorspringende Falte oder Platte, die entweder nur als ein cranialer Abschnitt vorhanden ist (Crista anthel. anterior Schwalbe) und erst durch eine während des Ohrschlusses auftretende Anthelixstammfalte ergänzt wird, oder als Stamm der Anthelix sich bis zur Plica antitragica erstreckt. In allen Fällen ist die Möglichkeit vorhanden, dass die Anthelix, gleichgültig wie sie gestaltet ist, durch Nuskelwirkung zusammengedrückt und dem Conchaboden genähert oder angelegt wird und auf solche Weise den Conchaeingang verengert resp. verschliesst. - Die Incisura intertragica, Tragus und Antitragus stellen eine Einrichtung vor, durch die das Cavum conchae abgeschlossen werden kann. - Die Hautfalte am dorsalen Rande des Porus wird benutzt, denselbew zu verengern. - Borstenähnliche, in bestimmter Weise angeordnete Haare scheinen das Eindringen des Wassers zu erschweren. - Die eingefettete haarlose Oberfläche der Concha nimmt das Wasser schwer an, verhindert es am Eindringen und lässt etwa eingedrungenes Wasser zu Tropfen zusammenlaufen, sodass es leicht abgeschüttelt werden kann. Denselben Zweck hat die dichte Behaarung des Innern der Ohmmuschel, wie sie z. B. der Bieber, der Fischotter und das Coypu aufweist. Dabei spielt wohl die zwischen den Haaren vorhandene Luft eine Rolle, wie sie auch bei der Körperbehaarung das Eindringen des Wassers bis auf die Haut verhindert. Dies lässt sich z. B. bei der Wasserspitzmaus und beim Myopotamus beobachten. 
Wenn wir sehen, dass an der Ohrmuschel vieler Süuger bestimmte Teile beim Schliessen der Ohrmuschel immer wieder in derselben Weise funktionieren, und wenn diese Teile uns in ihrem Vorhandensein und Gestalt erst durch ihre Funktion verständlich werden, so dürten wir wohl annehmen, dass in solchen Fällen, wo die gedachten Teile vorhanden sind, aber nicht funktionieren, diese ehemals funktioniert habeu, dann aber die Fähigkeit hierzu infolge von Nichtgebrauch oder von einseitiger Ausbildung der Ohrifuschel als Schallfänger verloren haben. So spricht die Tatsache, dass viele Tiere nicht im Stande sind, ihre Ohren zu schliessen, nicht gegen unsere Theorie. Übrigens ist es sicher, dass eine ganze Reihe von den Tieren, die wir in den zoologischen Gärten mit negativem Resultat geprüft haben, ein positives geben würden, wem man sie genauer untersuchen kömte.

Es ist klar, dass die komplizierte Gestalt der menschlichen Ohrmuschel durch ihre Funktion als Schalltrichter durchaus nicht erklärt wird. Dass die menschliche Ohrmuschel in mehrfacher Beziehung Rückbildungen aufweist, wird allgemein angenommen. Dies gilt für die Muschel als Ganzes wie für die Muskeln. Dass die Muskeln, sowohl die aus der Nachbarschaft zur Muschel ziehenden wie die Eigenmuskeln derselben rudimentär sind, geht aus ihrer Variabilität, ihrer schwachen Ausbildung wie aus ihrer geringen Funktionsfähigkeit hervor. Die ersteren Muskeln lassen erkennen, dass die Ohrmuschel einst als Ganzes bewegt werden konnte, wie dies bei vielen Tieren der Fall ist und dies noch jetzt manche Menschen vermögen. Es geschah dies vor allem beim Gebrauch der Ohrmuschel als Schalltrichter. Die Eigenmuskeln müssen in irgend einer Weise die Form der Ohrmuschel selbst verändert haben. Weiter weist Schwalbe (1889 1 . c. 208) darauf hin, dass in der Verschmelzung ursprünglich getrennter Koorpelstücke zum Gehörgangsknorpel eine Rückbildungserscheinung vorliege, wie auch die Spina helicis ein rudimentäres Scutulum rorstelle. 
Dass Reduktionsvorgänge nicht, wie man angenommen hat, die ganze Ohrmuschel, sondern nur die Anthelix und die freie Ohrfalte, die durch die Anthelix und den freien Ohrrand begrenzt wird, betroffen haben, hat Schwalbe (1889 z. B. p. 252) ausführlich erörtert. Für die Ohrfalte spreche die Verkleinerung und Einrollung, für die Anthelix die stärkere Ausbildung, die mit ersterer Erscheinung Hand in Hand gehe. Wir möchten diese Angabe noch mehr einschränken und nur für die Fossa scaphoidea und Helix als erwiesen ansehen, also für den Teil der Ohrmuschel, den wir als Scapha bezeichnen. Die Anthelix braucht sich, wie aus unserer Üntersuchung hervorgeht, in Grösse und Gestalt nur wenig anders verhalten $\mathrm{zu}$ haben, um funktionsfähig gewesen zu sein. Auch ist die Anthelix bei gut schlussfähigen Tierohren oft nicht viel anders gestaltet, wie beim Menschen.

Für die Reduktion der menschlichen Scapha spricht dagegen die relativ bessere Ausbildung beim Embryo und die nicht selten zu beobachtende stärkere Ausbildung beim Erwachsenen wie bei der Macacusform des menschlichen Ohres, die nur als atavistische und nicht etwa als progressive Form aufgefasst werden kann.

Die Tatsache, dass an der Ohrmuschel Rückbildungsvorgänge stattgefunden haben, erklärt uns aber noch nicht ihre Gestalt. Es wird jene ebenso wie die vieler Säuger erst verständljch, wenn wir annehmen, dass sie auf eine Form zurückgeht, die die Fähigkeit besessen hat, sich zu schliessen. Wir können uns nur so das Torhandensein und die Gestalt der Anthelix erklären. Dass diese, wie $\mathrm{Mach}$ meint, die Aufgabe habe, als Stütze zu wirken, hat schon Schwalbe zurückgewiesen (1889 p. 242). Für einen Schalltrichter wäre sie durchaus nicht nötig, wie sie ja auch vollständig fehlen kann, z. B. beim Kaninchen (Henneberg l. c. p. 135). Vielmehr hat die Anthelix einst, indem sie, schärfer hervortretend, eine Schlussfalte bildete, sich 
zusammenbog und sich gegen den Conchaboden legte, am Ohrschluss teilgenommen. Der Tragus und Antitragus haben, indem sie sich einander näherten und die Incisura intertragica schlossen und indem sie sich dem Conchaboden näherten, das Carum abgesperrt. Die Helix von der Helix ascendens an bis zum Tuberkulum Darwini, dem Homologon der Ohrspitze vieler Tiere, hat einst beim Scaphaschluss mitgewirkt, wie sie dies noch jetzt bei vielen 'Tieren tut. Der übrige Teil der menschlichen Helix stellt zusammen mit dem von ihm begrenzten Teil der Fossa scaphoidea eine reduzierte Scapha vor.

Auch Vorhandenseịn und Anordnung der kleinen Muskeln an der Ohrmuschel des Menschen geben ein Zeugnis von der ehemaligen Fähigkeit der Ohrmuschel, sich zu schliessen. Deutlich tritt dies an dem Musculus tragicus und antitragicus hervor. Jch schliesse mich hier Tataroff (1. c. p. 503) an, der diese beiden Muskeln Teilen des von Ruge bei den Halbaffen beschriebenen M. trago-antitragicus gleichsetzt. Wie jener einen Orbicularis auriculae vorstellt, der durch den M. tragohelicinus vervollständigt wird, so darf man auch annehmen, dass jene Muskeln in der Entwicklungsreihe des Menschen zu einer Zeit, da sie noch besser ausgebildet waren, die Wirkung eines Orbicularis gehabt haben. Sie haben nämlich die Incisura intertragica geschłossen, und der M. tragohelicinus hat wohl auch den Tragus dem Cavumboden genähert, sodass er sich deckelartig auf den Porus gelegt hat. Ebenso wird unsere Ansicht durch die Anordnung des M. obliquus und transversus auriculae unterstützt. Bei ihrer Kontraktion wurde die Eminentia fossae triangularis und die Eminentia scaphae der Eminentia conchae genähert. Dadurch trat aber die Anthelix auf der Innenseite - lateralen Seite der Ohrmuschel desto schärfer hervor und verkleinerte den Conchaeingang, wie dies beim Anthelixschluss der Tiere jetzt noch geschieht. Voraussetzung dabei ist, dass bei jener Ohrform der First der Anthelix nicht in der Weise lateral ge- 
richtet war, wie dies jetzt bei den meisten menschlichen Ohren der Fall ist, sondern nach vorn zu, nach der Concha hin. wie man dies noch jetzt bei manchen Menschen in ausgesprochener Weise findet. Ebenso kann man sich vorstellen, dass der M. helicis major beim Scaphaschluss wirksam gewesen ist.

Dass übrigens beim Menschen während des Tauchens das Wasser trotz mangelnden Ohrschlusses nicht in den Gehörgang läuft, hat seinen Grund darin, dass letzterer winklig geknickt ist, wodurch beim Untertauchen die Luft in ihm desto sicherer festgehalten wird, die dann das Eindringen des Wassers rerhindert. Vielleicht spielen hierbei oder haben hierbei die im äusseren Gehörgang stehenden Haare und die Einfettung durch das Cerumen eine Rolle gespielt. Bringt man die Ohren allmählich oder in schräger Stellung ins Wasser, so kamn die Luft entweichen und das Wasser dringt ein.

Aus dieser Betrachtungsweise ergeben sich neue Gesichtspunkte für Beobachtungen am menschlichen Ohr. So wären z. B. diejenigen Formen aufzusuchen, die einer schliessfähigen Ohrmuschel am nächsten kommen. Von solchen kann man annehmen, dass sie eine alte Form der Ohrmuschel repräsentieren. Auch die Eigenmuskeln der Muschel wären auf ihre Variabilität und auf ihre ehemalige Funktion von unserem Standpunkt aus näher zu untersuchen. Dabei dürfte sich herausstellen, dass die genannten Muskeln zuweilen noch kontraktionsfähig sind.

Eine ganze Anzahl von Fragen, die sich bei dieser Untersuchung aufdrängten, sind nicht einmal erwähnt worden. Die Gefahr, sich dabei in Spekulationen zu verlieren, liegt nahe. Weitere Beobachtungen sind hier nötig. 


\section{Literatur - Verzeichnis.}

1. Boenninghaus, G., Das Ohr des Zahnwales etc. Zoolog. Jahrbücher. Abt. f. Anatomie u. Ontogenie, 19. Bd., 1904.

2. Henneberg, B., Beiträge zur Entwickelung der Ohrmuschel. Anatomische Hefte, Bd. 36., 1908.

3. Schmidt, Joh., Vergleichend-anatomische Untersuchungen über die Ohrmuschel verschiedener Säugetiere. Inaug. Diss. Leipzig, 1902.

4. Schwalbe, G.. Inwiefern ist die menschliche Ohrmuschel ein rudimentäres Organ? Arch. f. Anat. u. Phys. Anat. Abt. Suppl. Jahrg., 1889.

5. Schwalbe, G., Das äussere Ohr. 6. Lieferung des Handbuches der Anatomie des Menschen. Herausgegeben von K. v. Bardeleben. Bd. 5, 2, Jena 1898.

6. Tatar off, D., Uber die Muskeln der Ohrmuschel und einige Besonderheiten des Ohrknorpels. Arch. f. Anat. u. Phys., Anat. Abt., Jahrg. 1887. 


\title{
Figuren-Erklärung
}

\author{
zu Tafel 7 u. 8.
}

Ohrmuschel des Hamsters (grösstenteils geschoren).

Fig. 1: offen, fast $11 / 2$ mal vergrössert.

Fig. 2: halbgeschlossen, etwas vergrössert.

Fig. 3: ganz geschlossen, etwas vergrössert.

\section{Ohrmuschel der Wanderratte.}

Fig. 4: offen, 2 mal vergrössert.

Fig. 5: geschlossen, doch ist der orale Teil der Scapha wieder aufgeklappt.

a) erster Modus, 2 mal vergrössert.

b) zweiter Modus, 2 mal vergrössert.

Fig. 6: vollständig geschlossen, 2 mal vergrössert.

Ohrmuschel der Waldmaus.

Fig. 7 : offen, 2 mal vergrössert.

Fig. 8: geschlossen, 2 mal vergrössert.

Fig. 9: geschlossen, doch hat sich der orale Teil der Scapha nicht herüber geklappt. 21/2 mal vergrössert.

Ohrmuschel des Meerschweinchens.

Fig. 10: offen (geschoren), etwas vergrössert.

Fig. 11: geschlossen, etwas vergrössert.

a) mit Behaarung, etwas vergrössert.

b) grösstenteils geschoren, etwas vergrössert.

Fig. 12: geschlossen, doch ist der orale Teil der Scapha aufgeklappt. geschoren.

a) erster Modus, etwas vergrössert.

b) zweiter Modus, etwas verkleinert. 


\title{
Erklärung der Abkürzangen.
}

\author{
a. $\cdots$ : Anthelix. \\ Ast. - Anthelixstamm-Falte. \\ c. s. = Crus anthelicis superius. \\ c. i. - Crus anthelicis inferius. \\ cr. a. = Crista anthel. anterior. \\ trg. - Tragus. \\ antrg. - Antitragus. \\ i. = Incisura intertragica. \\ pl. a. = Plica antitragica. \\ pl. l. = Plica longitudinalis. \\ o. sc. = oraler Abschnitt der Scapha. \\ ab. sc. = aboraler Abschnitt der Scapha. \\ pr. Qu. = proximale Querfalte der Scapha. \\ d. Qu. = distale Querfalte der Scapha. \\ W. - Wulst am Boden der Concha.
}

